Homology, Homotopy and Applications, vol.17(ד), 2015, pp.339-376

\title{
HOMOLOGICAL ALGEBRA FOR DIFFEOLOGICAL VECTOR SPACES
}

\author{
ENXIN WU \\ (communicated by Nathalie Wahl)
}

\begin{abstract}
Diffeological spaces are natural generalizations of smooth manifolds, introduced by J.M. Souriau and his mathematical group in the 1980's. As vector spaces, diffeological vector spaces appear canonically from geometry and analysis, and they also contain smooth information. In this paper, we first explore the basic algebraic and categorical constructions on diffeological vector spaces. Then we observe that not every short exact sequence of diffeological vector spaces splits. Motivated by this, we develop the natural analogues of basic tools of classical homological algebra by identifying a good class of projective objects in the category of diffeological vector spaces, together with some applications in analysis. Finally, we prove that there is a cofibrantly generated model structure on the category of diffeological chain complexes.
\end{abstract}

\section{Introduction}

The concept of diffeological space and the terminology were formulated by J.M. Souriau and his mathematical group in the 1980's ([S01], So2]) as follows:

Definition 1.1. A diffeological space is a set $X$ together with a specified set $\mathcal{D}_{X}$ of functions $U \rightarrow X$ (called plots) for every open set $U$ in $\mathbb{R}^{n}$ and for each $n \in \mathbb{N}$, such that for all open subsets $U \subseteq \mathbb{R}^{n}$ and $V \subseteq \mathbb{R}^{m}$ :

1. (Covering) Every constant map $U \rightarrow X$ is a plot.

2. (Smooth compatibility) If $U \rightarrow X$ is a plot and $V \rightarrow U$ is smooth, then the composition $V \rightarrow U \rightarrow X$ is also a plot.

3. (Sheaf condition) If $U=\cup_{i} U_{i}$ is an open covering and $U \rightarrow X$ is a set map such that each restriction $U_{i} \rightarrow X$ is a plot, then $U \rightarrow X$ is a plot.

We usually use the underlying set $X$ to represent the diffeological space $\left(X, \mathcal{D}_{X}\right)$.

Comparing it with the concept of a smooth manifold, a diffeological space starts with a set instead of a topological space, and it uses all open subsets of Euclidean

Received October 1, 2014, revised December 12, 2014; published on May 18, 2015.

2010 Mathematics Subject Classification: 18G25 (primary), 57P99, 26E10 (secondary).

Key words and phrases: Diffeological vector space, linear subduction, short exact sequence.

Article available at http://dx.doi.org/10.4310/HHA.2015.v17.n1.a17

Copyright (C) 2015, Enxin Wu. Permission to copy for private use granted. 
spaces for characterizing smoothness, subject to the above three axioms. It is a sheaf over a certain site and has an underlying set. This makes it a concrete sheaf; see [BB]].

The theory of diffeological spaces was further developed by several mathematicians, especially Souriau's students P. Donato and P. Iglesias-Zemmour. Recently, P. Iglesias-Zemmour published the book [[3]]. We refer the reader unfamiliar with diffeological spaces to [[-3] for terminology and details. For a three-page introduction to diffeological spaces, we recommend [CSW, Section 2]. Let us mention a few basic properties:

A smooth map between diffeological spaces is a function sending each plot of the domain to a plot of the codomain. Diffeological spaces with smooth maps form a category, denoted by $\mathfrak{D i f f}$. The category $\mathfrak{D}$ iff contains the category of smooth manifolds and smooth maps as a full subcategory. That is, every smooth manifold is automatically a diffeological space, and smooth maps between smooth manifolds in this new sense are the same as smooth maps between smooth manifolds in the usual sense. Moreover, the category $\mathfrak{D}$ iff is complete, cocomplete, and (locally) cartesian closed. Every (co)limit in Diff has the corresponding (co)limit in Set as the underlying set. For any diffeological spaces $X$ and $Y$, we write $C^{\infty}(X, Y)$ for the set of all smooth maps $X \rightarrow Y$. There is a natural diffeology (called the functional diffeology) on $C^{\infty}(X, Y)$ making it a diffeological space. Also, every diffeological space has a natural topology called the $\boldsymbol{D}$-topology, which is the same as the usual topology for every smooth manifold. See [1/3], Chapters 1 and 2] for more details.

Vector spaces are fundamental objects in mathematics. There are corresponding objects in diffeology called diffeological vector spaces; that is, they are both diffeological spaces and vector spaces such that addition and scalar multiplication maps are both smooth. P. Iglesias-Zemmour focused on fine diffeological vector spaces and used them to model some infinite dimensional spaces in [[1], [13]. K. Costello and O. Gwilliam used a class of diffeological vector spaces called differentiable vector spaces in their work on factorization algebras in quantum field theory in their book [CG]. Diffeological vector spaces also arise naturally in the study of tangent spaces of diffeological spaces; see, for example, [CW2]. The first goal of this paper is to study the basic categorical and algebraic properties of (fine) diffeological vector spaces, which are done in Sections $\mathbf{2}$, 国, and $\mathbf{6}$.

The main part of this paper is motivated by the following example from Section $\mathbb{\text { G. }}$. Let

$$
\phi: C^{\infty}(\mathbb{R}, \mathbb{R}) \rightarrow \prod_{\omega} \mathbb{R}
$$

be the map from the space of all smooth maps $\mathbb{R} \rightarrow \mathbb{R}$ to the product of countably many copies of $\mathbb{R}$ defined by $f \mapsto\left(f(0), f^{\prime}(0), f^{\prime \prime}(0), \ldots\right)$. It is clearly a linear map. Borel's lemma says that it is surjective. Let $K$ be its kernel. Then the classical homological algebra tells us that the short exact sequence

$$
0 \longrightarrow K \longrightarrow C^{\infty}(\mathbb{R}, \mathbb{R}) \stackrel{\phi}{\longrightarrow} \prod_{\omega} \mathbb{R} \longrightarrow 0
$$

splits as vector spaces. We prove that this is a short exact sequence of diffeological vector spaces in the sense of Definition [.5.5, which does not split smoothly; see Example 4.3 for details. As a corollary, we get a generalized version of Borel's lemma. 
Then we discuss the question of how to characterize the (non-)splitting of short exact sequences of diffeological vector spaces. One observes that the category $\mathfrak{D V e c t}$

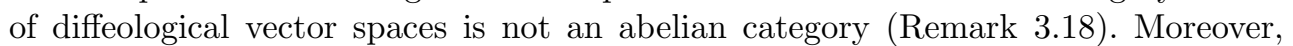
although the category $\mathfrak{D i f f}$ of diffeological spaces is equivalent to the category of concrete sheaves over a concrete site $[\mathbf{B H}]$, we have enough projectives (in the sense of Definition $[$ ] $)$ on the category $\mathfrak{D V e c t . ~ W e ~ d i s c u s s ~ t h e ~ b a s i c ~ e x a m p l e s ~ a n d ~ p r o p e r t i e s ~ o f ~}$ these projective diffeological vector spaces in Section [ [, and observe that they behave slightly differently from the usual projective modules over a ring. We develop basic tools of homological algebra for diffeological vector spaces using these projectives: Schanuel's lemma, the Horseshoe Lemma, the (Short) Five Lemma, etc. in Section $\boldsymbol{\square}$, and reach the fact that Ext $^{1}$ classifies short exact sequences of diffeological vector spaces up to equivalence.

Finally, in Section $\boldsymbol{\nabla}$, we prove that there is a cofibrantly generated model structure on the category of diffeological chain complexes. A projective resolution is then a cofibrant replacement under this model structure, and Ext* can be expressed using the hom-functor in the corresponding homotopy category, as expected.

Here is a detailed summary of the main results of this paper:

1. The category of diffeological vector spaces and smooth linear maps is complete

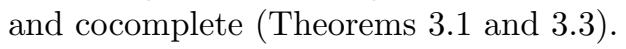

2. For every diffeological space, there is a free diffeological vector space generated by it, together with a universal property (Proposition 3.5 . Every diffeological vector space is a quotient vector space of a free diffeological vector space (Corollary [.53), but not every diffeological vector space is a free diffeological vector space generated by a diffeological space (Example [.]).

3. We define tensor products and duals for diffeological vector spaces in Section [1].

4. We define short exact sequences of diffeological vector spaces (Definition [.]. and we have necessary and sufficient conditions for when a short exact sequence of diffeological vector spaces splits (Theorem [.]6). Unlike the case of vector spaces, not every short exact sequence of diffeological vector spaces splits (Example 4.3). We also get a generalized version of Borel's lemma (Remark 4.5) as an application.

5. Fine diffeological vector spaces (Definition 5 ) behave like vector spaces, except for taking infinite products and duals (Examples $\$$ and 5 . Every fine diffeological vector space is a free diffeological vector space generated by a discrete diffeological space (Property (ד) ). A free diffeological vector space generated by a diffeological space is fine if and only if this diffeological space is discrete (Theorem 5.3).

6. Every fine diffeological vector space is Frölicher (Proposition 5.7 ).

7. We define projective diffeological vector spaces as projective objects in the category of diffeological vector spaces with respect to linear subductions (Definition [.]. ), and we have many equivalent characterizations (Propositions

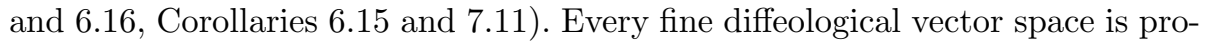
jective, as is every free diffeological vector space generated by a smooth manifold (Corollaries 6.3 and 6.4 ). But not every (free) diffeological vector space is projective (Example $\mathbf{6 . 9}$ ). However, there are enough projectives in the category of 
diffeological vector spaces (Theorem [.]3).

8. We have many interesting (non-)examples of (fine or projective) diffeological

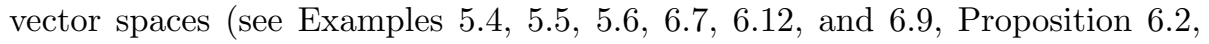
Corollaries [.3.] and [.4, and Remark [.8).

9. We establish basic tools of homological algebra for general diffeological vector spaces in Section $\mathbf{\square}$. As usual, every diffeological vector space has a diffeological projective resolution, and any two diffeological projective resolutions are diffeologically chain homotopic (see the paragraph before Lemma $\mathbf{Z} \boldsymbol{2})$. Schanuel's lemma, the Horseshoe Lemma, and the (Short) Five Lemma still hold in this

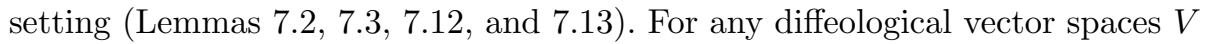
and $W$, we can define diffeological vector spaces $\operatorname{Ext}^{n}(V, W)$ (Definition 7.5 ). For any short exact sequence of diffeological vector spaces in the first or second variable, we get a long sequence of such diffeological vector spaces which is exact in the category $\mathfrak{V}$ ect of vector spaces and linear maps (Theorems $\mathbb{R . 8}$

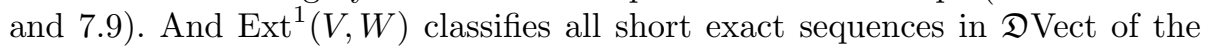
form $0 \rightarrow W \rightarrow A \rightarrow V \rightarrow 0$ up to equivalence (Theorem [द. 4).

10. There is a cofibrantly generated model structure on the category of diffeological chain complexes (Theorem 6 ). A diffeological projective resolution of a diffeological vector space $V$ is then a cofibrant replacement of the canonical diffeological chain complex $S^{0}(V)$ under this model structure (the paragraph after the proof of Lemma 6.3$)$, and $\operatorname{Ext}^{n}(V, W)$ can be expressed as $\mathrm{Ho}(\mathfrak{D C h})\left(S^{0}(V), S^{n}(W)\right)$ (Proposition 区.

\section{Acknowledgments}

I'd like to thank J.D. Christensen for very helpful discussions of some material in this paper, G. Sinnamon for the analytic proof of Example 5. $\mathbf{5}$, and the referee for many comments that led to great improvements in the exposition and for the suggestion of writing up the last section of this paper.

Convention: Throughout this paper, unless otherwise specified,

- every vector space is over the field $\mathbb{R}$ of real numbers;

- every linear map is over $\mathbb{R}$;

- every tensor product is over $\mathbb{R}$, i.e., $\otimes=\otimes_{\mathbb{R}}$;

- every smooth manifold is assumed to be Hausdorff, second-countable, finite dimensional, and without boundary;

- every smooth manifold is equipped with the standard diffeology, and in particular, $\mathbb{R}$ has the standard diffeology when viewed as a diffeological space;

- every subset of a diffeological space is equipped with the sub-diffeology;

- every product of diffeological spaces is equipped with the product diffeology;

- every quotient set of a diffeological space is equipped with the quotient diffeology, i.e., a plot of a quotient space is locally the quotient map composed with a plot of the total space; 
- every function space of diffeological spaces is equipped with the functional diffeology;

- it is known ([1]3]) that for a given set $X$, the set of all diffeologies on $X$ with inclusions forms a complete lattice; we call a diffeology $\mathcal{D}$ on $X$ with certain properties the smallest, if every diffeology on $X$ with these properties contains $\mathcal{D}$;

- an induction $i: X \rightarrow Y$ between diffeological spaces is a smooth injective map such that $X$ is diffeomorphic (i.e., isomorphic in the category $\mathfrak{D}$ iff) to the image $i(X)$ equipped with the sub-diffeology of $Y$.

\section{Definition and basic examples}

In this section, we recall the definitions of diffeological vector spaces and smooth linear maps between them, together with some basic examples.

Definition 2.1. A diffeological vector space is a vector space $V$ together with a diffeology, such that the addition map $V \times V \rightarrow V$ and the scalar multiplication map $\mathbb{R} \times V \rightarrow V$ are both smooth.

Definition 2.2. A smooth linear map between two diffeological vector spaces is a function which is both smooth and linear.

Diffeological vector spaces with smooth linear maps form a category, denoted by DVect.

It is straightforward to verify the following examples:

Example 2.3. Every vector space with the indiscrete diffeology is a diffeological vector space. We write $\mathbb{R}_{\text {ind }}$ for the diffeological vector space with the underlying set $\mathbb{R}$ and the indiscrete diffeology. It will be used to construct many (counter)examples in the following sections.

Example 2.4. Every linear subspace of a diffeological vector space is a diffeological vector space.

Example 2.5. Every product of diffeological vector spaces is a diffeological vector space. In particular, $\prod_{\omega} \mathbb{R}$, the product of countably many copies of $\mathbb{R}$, is a diffeological vector space. It will be one of the most important diffeological vector spaces in this paper.

Example 2.6. Every quotient vector space of a diffeological vector space is a diffeological vector space.

Example 2.7. Let $X$ be a diffeological space, and let $V$ be a diffeological vector space. Then $C^{\infty}(X, V)$ with pointwise addition and pointwise scalar multiplication is a diffeological vector space. Moreover, the map

$$
m: C^{\infty}(X, \mathbb{R}) \times C^{\infty}(X, V) \rightarrow C^{\infty}(X, V) \text { with } m(f, g)(x)=f(x) g(x)
$$

is a well-defined smooth map. 
Example 2.8. The $D$-topology on diffeological spaces induces a functor $D: \mathfrak{D i f f} \rightarrow$ Top, which has a right adjoint $C:$ Top $\rightarrow$ Diff sending a topological space to the space with the same underlying set and plots all continuous maps; see CSW, Proposition 3.3]. It is straightforward to check that every topological vector space with the continuous diffeology is a diffeological vector space. Moreover, if we write TVect for the category of topological vector spaces and continuous linear maps, then the adjoint pair

$$
D: \mathfrak{D i f f} \rightleftharpoons \mathfrak{T} \text { op }: C
$$

induces an adjoint pair

$$
D: \mathfrak{D V e c t} \rightleftharpoons \mathfrak{T} \text { Vect }: C \text {. }
$$

Now we introduce one of the most important concepts in this paper. Recall that a smooth map $f: X \rightarrow Y$ between diffeological spaces is a subduction if $Y$ is diffeomorphic to the quotient space $X / \sim$, where $x \sim x^{\prime}$ if $f(x)=f\left(x^{\prime}\right)$. We call a map $g: V \rightarrow W$ between diffeological vector spaces a linear subduction if it is both linear and a subduction.

We will show the most important property for linear subduction in Corollary 6.5 that every plot of the codomain of a linear subduction globally lifts to a plot of the domain, which is clearly not true for a general subduction. This is one of the key ingredients for developing homological algebra of diffeological vector spaces.

\section{Categorical properties}

In this section, we study the categorical properties of the category $\mathfrak{D V e c t}$ of diffeo-

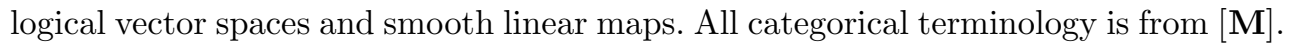
We begin by showing that $\mathfrak{D}$ Vect is complete and cocomplete. Then we focus on the algebraic aspects of diffeological vector spaces. We construct the free diffeological vector space generated by an arbitrary diffeological space, and define tensor products, duals, and short exact sequences of diffeological vector spaces. We also discuss some isomorphism theorems and necessary and sufficient conditions for when a short exact sequence of diffeological vector spaces splits in $\mathfrak{D V e c t . ~ U n l i k e ~ t h e ~ c a s e ~ o f ~ v e c - ~}$ tor spaces, we will see in the next section that not every short exact sequence of diffeological vector spaces splits in $\mathfrak{D}$ Vect.

Theorem 3.1. The category $\mathfrak{D}$ Vect is complete.

Proof. Every limit can be constructed via equalizers and products, so Examples प. and 5 show that we have every limit.

The underlying set of a coproduct in $\mathfrak{D}$ iff is the disjoint union of the underlying sets, so a coproduct in $\mathfrak{D i f f}$ cannot be the coproduct in $\mathfrak{D}$ Vect. On the other hand, the forgetful functor $\mathfrak{D V e c t} \rightarrow \mathfrak{V}$ ect is a left adjoint; see Theorem $\mathbf{D}$. So if a coproduct exists in $\mathfrak{D V e c t}$, the underlying set must be the direct sum of the underlying vector spaces. The proof of the following proposition tells us how to put a suitable diffeology on this set to make it a coproduct in $\mathfrak{D V}$ ect.

Proposition 3.2. The category $\mathfrak{D V e c t ~ h a s ~ a r b i t r a r y ~ c o p r o d u c t s . ~}$ 
Proof. Let $\left\{V_{i}\right\}_{i \in I}$ be a set of diffeological vector spaces. We claim that the set $V=\oplus_{i \in I} V_{i}$ with the final diffeology for all canonical maps $\prod_{j \in J} V_{j} \rightarrow V$ and for all finite subsets $J$ of the index set $I$ is the colimit in $\mathfrak{D V e c t . ~ H e r e ~ i s ~ t h e ~ p r o o f . ~}$ For any diffeological vector space $W$ and any smooth linear maps $V_{i} \rightarrow W$, by the universal property of colimit in $\mathfrak{V}$ ect, there is a unique linear map $V \rightarrow W$ making the required diagrams commutative. It is then straightforward to check that each composite $\prod_{j \in J} V_{j} \rightarrow V \rightarrow W$ is smooth by definition of diffeological vector space. Therefore, $V$ is the colimit of $\left\{V_{i}\right\}_{i \in I}$ in $\mathfrak{D}$ Vect.

From now on, $\oplus_{i \in I} V_{i}$ is always equipped with the coproduct diffeology in $\mathfrak{D}$ Vect as introduced in the proof of the above proposition for a set $\left\{V_{i}\right\}_{i \in I}$ of diffeological vector spaces.

Theorem 3.3. The category $\mathfrak{D V e c t}$ is cocomplete.

Proof. Every colimit can be constructed via coequalizers and coproducts, so Example 20 and Proposition show that we have every colimit.

Now we discuss how to define hom-objects in $\mathfrak{D V e c t :}$

Given two diffeological vector spaces $V$ and $W$, we write $L^{\infty}(V, W)$ for the set of all smooth linear maps $V \rightarrow W$. Since $L^{\infty}(V, W)$ is a linear subspace of $C^{\infty}(V, W)$, by Examples $\left[.4\right.$ and $[],. L^{\infty}(V, W)$ with the sub-diffeology of $C^{\infty}(V, W)$ is a diffeological vector space. From now on, $L^{\infty}(V, W)$ is always equipped with this diffeology (called the functional diffeology) when viewed as a diffeological (vector) space. As an easy consequence, the evaluation map $V \times L^{\infty}(V, W) \rightarrow W$ is smooth.

Example 3.4. Let $V$ be a diffeological vector space. Then the map $L^{\infty}(\mathbb{R}, V) \rightarrow V$ defined by $f \mapsto f(1)$ is an isomorphism in $\mathfrak{D}$ Vect.

In order to define tensor products for diffeological vector spaces, we need the following proposition, which will be very useful throughout this paper.

Proposition 3.5. The forgetful functor $\mathfrak{D}$ Vect $\rightarrow$ Diff has a left adjoint.

Proof. Given a diffeological space $X$, write $F(X)$ for the free vector space generated by the underlying set of $X$. For $p_{1}: U_{1} \rightarrow X, \cdots, p_{n}: U_{n} \rightarrow X$ plots of $X$, write

$$
\left(\mathbb{R} \times U_{1}\right) \times \cdots \times\left(\mathbb{R} \times U_{n}\right) \rightarrow F(X)
$$

for the map defined by $\left(\left(r_{1}, u_{1}\right), \ldots,\left(r_{n}, u_{n}\right)\right) \mapsto r_{1}\left[p_{1}\left(u_{1}\right)\right]+\cdots+r_{n}\left[p_{n}\left(u_{n}\right)\right]$. Equip $F(X)$ with the diffeology generated by all such maps for all $n \in \mathbb{Z}^{+}$. It is clear that this is the smallest diffeology on $F(X)$ such that $F(X)$ is a diffeological vector space and the canonical map $i_{X}: X \rightarrow F(X)$ is smooth. We call $F(X)$ with this diffeology the free diffeological vector space generated by the diffeological space $X$. Moreover, we have the universal property that for any diffeological vector space $V$ and any smooth map $f: X \rightarrow V$, there exists a unique smooth linear map $g: F(X) \rightarrow V$ 
making the following triangle commutative:

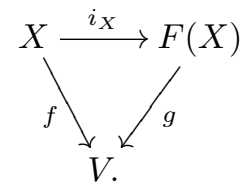

Therefore, we can define $F: \mathfrak{D i f f} \rightarrow \mathfrak{D V}$ ect by sending $X \rightarrow Y$ to the unique smooth linear map $F(X) \rightarrow F(Y)$ defined by the universal property. Then $F$ is a functor which is left adjoint to the forgetful functor $\mathfrak{D}$ Vect $\rightarrow \mathfrak{D}$ iff.

In particular, if $f: X \rightarrow Y$ is a subduction of diffeological spaces, then $F(f): F(X) \rightarrow$ $F(Y)$ is a linear subduction of (free) diffeological vector spaces.

Remark 3.6. The universal property of free diffeological vector spaces says that if $X$ is a diffeological space and $V$ is a diffeological vector space, then there is a natural bijection between $C^{\infty}(X, V)$ and $L^{\infty}(F(X), V)$. Indeed, this is an isomorphism in DVect.

Similarly, if $\left\{X_{i}\right\}_{i \in I}$ is a set of diffeological spaces and $\left\{V_{j}\right\}_{j \in J} \cup\{V\}$ is a set of diffeological vector spaces, then we have natural isomorphisms in $\mathfrak{D V e c t :}$

$$
\begin{gathered}
C^{\infty}\left(\coprod_{i \in I} X_{i}, V\right) \cong \prod_{i \in I} C^{\infty}\left(X_{i}, V\right), \\
L^{\infty}\left(V, \prod_{j \in J} V_{j}\right) \cong \prod_{j \in J} L^{\infty}\left(V, V_{j}\right),
\end{gathered}
$$

and

$$
L^{\infty}\left(\oplus_{j \in J} V_{j}, V\right) \cong \prod_{j \in J} L^{\infty}\left(V_{j}, V\right)
$$

Example 3.7. Not every diffeological vector space is a free diffeological vector space generated by a diffeological space. $\mathbb{R}_{\text {ind }}$ is such an example.

Now we discuss tensor products in $\mathfrak{D V}$ Vect:

Let $V$ and $W$ be two diffeological vector spaces. Since the vector space $V \otimes W$ is a quotient vector space of the free diffeological vector space $F(V \times W)$ generated by the product space $V \times W$, by Example [2], $V \otimes W$ is a diffeological vector space with the quotient diffeology. From now on, we always equip $V \otimes W$ with this diffeology.

As usual, we have the following adjoint pair:

Theorem 3.8. For any diffeological vector space $V$, there is an adjoint pair

$$
-\otimes V: \mathfrak{D V e c t} \rightleftharpoons \mathfrak{D V e c t}: L^{\infty}(V,-) .
$$

Proof. Let $W$ and $A$ be diffeological vector spaces. By definition of functional diffeology, one shows directly that a map $W \rightarrow L^{\infty}(V, A)$ is smooth linear if and only if the adjoint set map $W \times V \rightarrow A$ is smooth bilinear. By the universal property of free diffeological vector space generated by a diffeological space in the proof of Proposition 5.5 , one then shows that a map $W \times V \rightarrow A$ is smooth bilinear if and only if the induced map $W \otimes V \rightarrow A$ is smooth linear. 
Here are some basic properties of tensor products of diffeological vector spaces.

\section{Remark 3.9.}

1. Given diffeological vector spaces $V_{1}, V_{2}$, and $V_{3}, V_{1} \otimes V_{2}$ is naturally isomorphic to $V_{2} \otimes V_{1}$ in $\mathfrak{D V e c t}$, and $\left(V_{1} \otimes V_{2}\right) \otimes V_{3}$ is naturally isomorphic to $V_{1} \otimes\left(V_{2} \otimes\right.$ $\left.V_{3}\right)$ in $\mathfrak{D V e c t}$. The second isomorphism is indicated by the fact that the canonical projection map from $F\left(V_{1} \times V_{2} \times V_{3}\right)$ to either $\left(V_{1} \otimes V_{2}\right) \otimes V_{3}$ or $V_{1} \otimes\left(V_{2} \otimes V_{3}\right)$ is a (linear) subduction.

2. Given a set of diffeological vector spaces $\left\{V_{i}\right\}_{i \in I} \cup\{W\},\left(\oplus_{i \in I} V_{i}\right) \otimes W$ is isomorphic to $\oplus_{i \in I}\left(V_{i} \otimes W\right)$ in $\mathfrak{D V e c t .}$

3. For any diffeological vector space $V$, the map $V \rightarrow V \otimes \mathbb{R}$ defined by $v \mapsto v \otimes 1$ is an isomorphism in $\mathfrak{D}$ Vect.

Proposition 3.10. Let $X$ and $Y$ be diffeological spaces. Then $F(X \times Y)$ is isomorphic to $F(X) \otimes F(Y)$ in $\mathfrak{D V}$ ect.

Proof. Since $i_{X}: X \rightarrow F(X)$ and $i_{Y}: Y \rightarrow F(Y)$ are smooth, so is $i_{X} \times i_{Y}: X \times Y \rightarrow$ $F(X) \times F(Y)$ and hence the composite $i_{F(X) \times F(Y)} \circ\left(i_{X} \times i_{Y}\right): X \times Y \rightarrow F(F(X) \times$ $F(Y))$. The last map induces a smooth linear map $F(X \times Y) \rightarrow F(F(X) \times F(Y))$. So we get a smooth linear map $F(X \times Y) \rightarrow F(X) \otimes F(Y)$ given by $\sum c_{j}\left[x_{j}, y_{j}\right] \mapsto$ $\sum c_{j}\left[x_{j}\right] \otimes\left[y_{j}\right]$. It is known from general algebra that this map is an isomorphism in $\mathfrak{V}$ ect. Therefore, we are left to show that the inverse map is smooth. By definition of the diffeology on $F(X) \otimes F(Y)$, it is enough to show that the canonical projection map $F(F(X) \times F(Y)) \rightarrow F(X \times Y)$ given by $\sum_{i} a_{i}\left[\sum_{j} b_{i j}\left[x_{j}\right], \sum_{k} c_{i k}\left[y_{k}\right]\right] \mapsto$ $\sum_{i, j, k} a_{i} b_{i j} c_{i k}\left[x_{j}, y_{k}\right]$ is smooth. This follows directly from the description of the diffeology on the free diffeological vector space generated by a diffeological space in the proof of Proposition 5.5 .

Now we discuss the dual to a diffeological vector space:

Let $V$ be a diffeological vector space. Write $\tilde{D}(V)$ for the dual diffeological vector space $L^{\infty}(V, \mathbb{R})$. Then $\tilde{D}$ is a functor $\mathfrak{D V}$ Vect $\rightarrow \mathfrak{D V e c t}^{o p}$, and we have a natural transformation $1 \rightarrow \tilde{D}^{2}$ : $\mathfrak{D}$ Vect $\rightarrow \mathfrak{D}$ Vect.

Example 3.11. It is not true that for every finite dimensional diffeological vector space $V$, the canonical map $V \rightarrow \tilde{D}^{2}(V)$ is a diffeomorphism. And it is not true that for every diffeological vector space $V$, the canonical map $V \rightarrow \tilde{D}^{2}(V)$ is injective. For example, $\tilde{D}\left(\mathbb{R}_{\text {ind }}\right)=\mathbb{R}^{0}=\tilde{D}^{2}\left(\mathbb{R}_{\text {ind }}\right)$. On the other hand, the canonical map $V \rightarrow$ $\tilde{D}^{2}(V)$ is injective if and only if $\tilde{D}(V)$ separates points; that is, for any $v \neq v^{\prime} \in V$, there exists $l \in \tilde{D}(V)$ such that $l(v) \neq l\left(v^{\prime}\right)$.

Here are some isomorphism theorems:

\section{Proposition 3.12.}

1. Let $f: V \rightarrow W$ be a linear subduction between diffeological vector spaces. Then $\tilde{f}: V / \operatorname{ker}(f) \rightarrow W$ defined by $\tilde{f}(v+\operatorname{ker}(f))=f(v)$ is an isomorphism in $\mathfrak{D V e c t .}$

2. If $A$ is a diffeological vector space, $B$ is a linear subspace of $A$, and $C$ is a linear subspace of $B$, then $(A / C) /(B / C)$ is isomorphic to $A / B$ in $\mathfrak{D V e c t .}$

Proof. This is easy. 
Now the following result is clear:

Corollary 3.13. Every diffeological vector space is isomorphic to a quotient vector space of a free diffeological vector space in $\mathfrak{D V e c t .}$

Proof. Let $V$ be a diffeological vector space. Then the smooth map $1_{V}: V \rightarrow V$ induces a smooth linear map $\eta: F(V) \rightarrow V$ such that $\eta \circ i_{V}=1_{V}$, where $i_{V}: V \rightarrow$ $F(V)$ is the canonical map. This equality implies that $\eta$ is a subduction. Therefore, $V$ is isomorphic to $F(V) / \operatorname{ker}(\eta)$ in $\mathfrak{D}$ Vect.

Remark 3.14. If $V$ is a diffeological vector space, then the canonical map $i_{V}: V \rightarrow$ $F(V)$ is smooth but not linear. Therefore, the short exact sequence $0 \rightarrow \operatorname{ker}(\eta) \rightarrow$ $F(V) \rightarrow V \rightarrow 0$ of diffeological vector spaces in the proof of the above corollary does not split smoothly in general; see Theorem $\mathbf{5 . T}$ and Example $\mathbf{6 . g}(2)$. When $V$ is projective (see Definition $[$. d), the above short exact sequence splits smoothly.

Definition 3.15. Let $A, V$, and $B$ be diffeological vector spaces. Let $i: A \rightarrow V$ and $p: V \rightarrow B$ be smooth linear maps. We say that

$$
0 \longrightarrow A \stackrel{i}{\longrightarrow} V \stackrel{p}{\longrightarrow} B \longrightarrow 0
$$

is a short exact sequence in $\mathfrak{D V e c t}$, if

1. it is a short exact sequence in $\mathfrak{V e c t}$;

2. $i$ is an induction and $p$ is a subduction.

We call $i$ a linear induction.

One can show easily that if $f: V \rightarrow W$ is a linear subduction between diffeological vector spaces, then $f^{*}: L^{\infty}(W, A) \rightarrow L^{\infty}(V, A)$ is a linear induction for any diffeological vector space $A$.

Theorem 3.16. Let

$$
0 \longrightarrow A \stackrel{i}{\longrightarrow} V \stackrel{p}{\longrightarrow} B \longrightarrow 0
$$

be a short exact sequence in $\mathfrak{D V e c t}$. The following are equivalent.

1. There exists a smooth linear map $r: V \rightarrow A$ such that $r \circ i=1_{A}$.

2. There exists a smooth linear map $q: B \rightarrow V$ such that $p \circ q=1_{B}$.

3. The short exact sequence is isomorphic to

$$
0 \longrightarrow A \stackrel{i_{1}}{\longrightarrow} A \times B \stackrel{p_{2}}{\longrightarrow} B \longrightarrow 0
$$

in $\mathfrak{D V e c t}$, with identity maps on $A$ and $B$. In particular, $V$ is isomorphic to $A \times B$ in $\mathfrak{D}$ Vect.

In this case, we say that the short exact sequence

$$
0 \longrightarrow A \stackrel{i}{\longrightarrow} V \stackrel{p}{\longrightarrow} B \longrightarrow 0
$$

in $\mathfrak{D V e c t}$ splits smoothly, and we call $A$ (and $B$ ) a smooth direct summand of $V$. We will show in Example 4.3 that not every short exact sequence in $\mathfrak{D}$ Vect splits smoothly. 
Proof. If we only consider the statements in $\mathfrak{V}$ ect instead of $\mathfrak{D V e c t}$, then this is a standard result from algebra; see [H], Theorem IV.1.18] for instance. We are left to prove the smoothness of certain maps.

$(3) \Rightarrow(1)$ and $(3) \Rightarrow(2)$ are clear.

$(1) \Rightarrow(3)$ : By [Hu, the Short Five Lemma 1.17], $(r, p): V \rightarrow A \times B$ is an isomorphism in $\mathfrak{V}$ ect. Its inverse can be written as $i+q: A \times B \rightarrow V$ for some linear map $q: B \rightarrow V$. It is straightforward to check that $q \circ p=1_{V}-i \circ r$. Hence, $q \circ p$ is smooth. Then $p$ being a subduction implies that $q$ is smooth. So $i+q$ is smooth. Therefore, $(r, p)$ is an isomorphism in $\mathfrak{D V e c t}$, which implies the isomorphism of the two short exact sequences in $\mathfrak{D V e c t}$.

$(2) \Rightarrow(3)$ can be proved dually as $(1) \Rightarrow(3)$.

Now the following result is straightforward:

Corollary 3.17. Let $V$ and $W$ be diffeological vector spaces.

1. Let $i: V \rightarrow W$ and $r: W \rightarrow V$ be smooth linear maps such that $r \circ i=1_{V}$. Then there exists a diffeological vector space $X$ such that $W$ is isomorphic to $V \times X$ in $\mathfrak{D V e c t}$.

2. Let $p: W \rightarrow V$ and $q: V \rightarrow W$ be smooth linear maps such that $p \circ q=1_{V}$. Then there exists a diffeological vector space $X$ such that $W$ is isomorphic to $V \times X$ in $\mathfrak{D V}$ ect.

Remark 3.18. It is easy to see that the category $\mathfrak{D}$ Vect is additive with kernels and cokernels. However, it is not abelian, since a morphism in $\mathfrak{D V e c t}$ is monic if and only if the underlying set map is injective, but not necessarily an induction. Indeed, $\mathfrak{D}$ Vect is a quasi-abelian category in the sense of [SC, Definition 1.1.3] with strict epimorphisms the linear subductions and strict monomorphisms the linear inductions.

Remark 3.19. M. Vincent discussed tensor products and duals of diffeological vector spaces in his masters thesis $[\mathbf{\nabla}$, Chapter 2]. He also showed that a vector space with a pre-diffeology (that is, a set of functions from open subsets of Euclidean spaces to this vector space) has a smallest diffeology containing the original pre-diffeology which makes it a diffeological vector space. This is more general than the construction of free diffeological vector space generated by a diffeological space in this section.

\section{More examples}

In this section, we present two more examples of diffeological vector spaces from analysis. These examples were introduced in the framework of Frölicher spaces in $[\mathbf{K M}]$, and we adapt the proofs to the diffeological setting. The second example is the main motivation for developing (relative) homological algebra for diffeological vector spaces. At the end, we also get a generalized version of Borel's lemma.

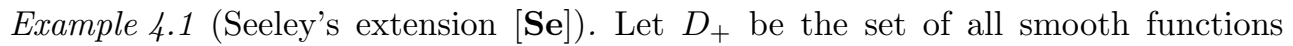

$f: \mathbb{R}^{n} \times \mathbb{R}^{>0} \rightarrow \mathbb{R}$ such that $\frac{\partial^{|m|} f}{\partial(x, t)^{m}}$ has continuous limits as $t \rightarrow 0$, for every $m \in$ $\mathbb{N}^{n+1}$. Then $D_{+}$is a linear subspace of $C^{\infty}\left(\mathbb{R}^{n} \times \mathbb{R}^{>0}, \mathbb{R}\right)$. Moreover, there is a smooth 
linear map

$$
E: D_{+} \rightarrow C^{\infty}\left(\mathbb{R}^{n} \times \mathbb{R}, \mathbb{R}\right)
$$

such that $E(f)(x, t)=f(x, t)$ when $t>0$.

\section{Remark 4.2.}

1. It is shown in $[\mathbf{S e}]$ that the map $E$ in the above example is continuous if both $D_{+}$and $C^{\infty}\left(\mathbb{R}^{n} \times \mathbb{R}, \mathbb{R}\right)$ are equipped with several topologies. However, it is straightforward to see that $E$ is actually smooth in the diffeological sense. In particular, $E$ is continuous if both $D_{+}$and $C^{\infty}\left(\mathbb{R}^{n} \times \mathbb{R}, \mathbb{R}\right)$ are equipped with the $D$-topology.

2. The inclusion map $i: \mathbb{R}^{n} \times \mathbb{R}^{>0} \hookrightarrow \mathbb{R}^{n} \times \mathbb{R}$ induces a smooth linear map

$$
i^{*}: C^{\infty}\left(\mathbb{R}^{n} \times \mathbb{R}, \mathbb{R}\right) \rightarrow C^{\infty}\left(\mathbb{R}^{n} \times \mathbb{R}^{>0}, \mathbb{R}\right) .
$$

It is clear that $\operatorname{Im}\left(i^{*}\right) \subseteq D_{+}$. By abuse of notation, we write $i^{*}: C^{\infty}\left(\mathbb{R}^{n} \times\right.$ $\mathbb{R}, \mathbb{R}) \rightarrow D_{+}$. Then $i^{*} \circ \bar{E}=1_{D_{+}}$. Therefore, $E$ is an induction. In particular, if we equip $C^{\infty}\left(\mathbb{R}^{n} \times \mathbb{R}, \mathbb{R}\right)$ with the $D$-topology, then the $D$-topology on $D_{+}$ is the initial topology with respect to the map $E$. Moreover, the $D$-topology on $C^{\infty}\left(\mathbb{R}^{n} \times \mathbb{R}, \mathbb{R}\right)$ is the weak topology [CSW , Corollary 4.10$]$, which is the same as "the topology of uniform convergence of each derivative on compact subsets of $\mathbb{R}^{n+1 "}[\mathbf{S E}]$.

3. Let

$$
F=\left\{f \in C^{\infty}\left(\mathbb{R}^{n} \times \mathbb{R}, \mathbb{R}\right) \mid i^{*}(f)=0 \in C^{\infty}\left(\mathbb{R}^{n} \times \mathbb{R}^{>0}, \mathbb{R}\right)\right\} .
$$

Then $F$ is a linear subspace of $C^{\infty}\left(\mathbb{R}^{n} \times \mathbb{R}, \mathbb{R}\right)$. Moreover, $C^{\infty}\left(\mathbb{R}^{n} \times \mathbb{R}, \mathbb{R}\right)$ is

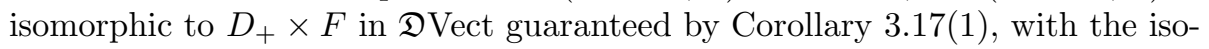
morphism given by

$$
C^{\infty}\left(\mathbb{R}^{n} \times \mathbb{R}, \mathbb{R}\right) \rightarrow D_{+} \times F, h \mapsto\left(i^{*}(h), h-E \circ i^{*}(h)\right)
$$

and

$$
D_{+} \times F \rightarrow C^{\infty}\left(\mathbb{R}^{n} \times \mathbb{R}, \mathbb{R}\right),(g, f) \mapsto E(g)+f .
$$

Example 4.3. Let

$$
\phi: C^{\infty}(\mathbb{R}, \mathbb{R}) \rightarrow \prod_{\omega} \mathbb{R} \text { be defined by }(\phi(f))_{n}=f^{(n)}(0) .
$$

Then $\phi$ is a smooth linear map with kernel

$$
K=\left\{f \in C^{\infty}(\mathbb{R}, \mathbb{R}) \mid f^{(n)}(0)=0 \text { for all } n \in \mathbb{N}\right\} .
$$

By Borel's lemma (a more general version will be proved in Claim 1 below), $\phi$ is surjective. Therefore, there is a smooth linear bijection

$$
\bar{\phi}: C^{\infty}(\mathbb{R}, \mathbb{R}) / K \rightarrow \prod_{\omega} \mathbb{R} .
$$

We will show through the following three claims that

$$
0 \longrightarrow K \longrightarrow C^{\infty}(\mathbb{R}, \mathbb{R}) \stackrel{\phi}{\longrightarrow} \prod_{\omega} \mathbb{R} \longrightarrow 0
$$

is a short exact sequence in $\mathfrak{D V e c t}$, which does not split smoothly. 
Remark 4.4. On the contrary, for any $n \in \mathbb{N}$, let $K_{n}=\left\{f \in C^{\infty}(\mathbb{R}, \mathbb{R}) \mid f^{(i)}(0)=\right.$ 0 for all $i \leqslant n\}$. Then the short exact sequence

$$
0 \longrightarrow K_{n} \longrightarrow C^{\infty}(\mathbb{R}, \mathbb{R}) \stackrel{\phi_{n}}{\longrightarrow} \prod_{n} \mathbb{R} \longrightarrow 0
$$

in $\mathfrak{D V e c t}$ does split smoothly, where $\phi_{n}(f)=\left(f(0), f^{\prime}(0), \ldots, f^{(n)}(0)\right)$.

Claim 1 (Generalized Borel's lemma, the local version): The map $\bar{\phi}$ is an isomorphism in $\mathfrak{D}$ Vect.

We are left to show that $\phi$ is a subduction. Let $F: U \rightarrow \prod_{\omega} \mathbb{R}$ be a plot. For any $x \in U$, fix two open neighborhoods $V$ and $V^{\prime}$ of $x$ in $U$, such that $V \subset \bar{V} \subset V^{\prime}$ with $\bar{V}$ compact. Fix $h \in C^{\infty}(\mathbb{R}, \mathbb{R})$ such that $h$ has compact support and $h(t)=t$ in an open neighborhood of 0 . Let

$$
\mu_{0}=2 \max \left\{1+\left\|F_{0}(x)\right\| \mid x \in V\right\}
$$

and let

$$
\mu_{n}=2 \max \left\{1+\mu_{n-1}\right\} \cup\left\{1+\left\|\frac{D^{\alpha} F_{n}(x)}{n !}\left(h^{n}\right)^{(j)}(t)\right\||x \in V,| \alpha \mid+j \leqslant n, t \in \mathbb{R}\right\} .
$$

Then $\left(\mu_{n}\right)$ is an increasing sequence with $\mu_{n} \geqslant 2 n+2$ for all $n \in \mathbb{N}$. Now we define

$$
\tilde{G}: V \times \mathbb{R} \rightarrow \mathbb{R} \text { by } \tilde{G}(x, t)=\sum_{m=0}^{\infty} \frac{F_{m}(x)}{m !}\left(\frac{h\left(t \mu_{m}\right)}{\mu_{m}}\right)^{m} .
$$

For any $t \neq 0$, this is a finite sum of smooth functions, so $\tilde{G}$ is smooth there. For $t$ near 0 , one can show by Weierstrass M-test that

$$
\sum_{m=0}^{\infty} \frac{D^{\alpha} F_{m}(x)}{m !}\left(\frac{\left(h\left(t \mu_{m}\right)^{m}\right)^{(j)}}{\mu_{m}^{m}}\right)
$$

is uniformly convergent for all $x \in V, \alpha \in \mathbb{N}^{\operatorname{dim}(U)}$, and $j \in \mathbb{N}$. So $\tilde{G}$ is also smooth at $t=0$, and hence $\tilde{G}$ is smooth on $V \times \mathbb{R}$. In other words, the adjoint map

$$
G: V \rightarrow C^{\infty}(\mathbb{R}, \mathbb{R})
$$

is smooth. Note that

$$
\tilde{G}(x, t) \sim \sum_{m=0}^{\infty} \frac{F_{m}(x)}{m !} t^{m} \text { as } t \rightarrow 0
$$

So

$$
(\phi \circ G)_{n}(x)=\frac{\partial^{n} \tilde{G}}{\partial t^{n}}(x, 0)=F_{n}(x)
$$

for all $x \in V$ and $n \in \mathbb{N}$. Therefore, $\phi$ is a subduction.

Claim 2: The $D$-topology on $\prod_{\omega} \mathbb{R}$ is the product topology.

Observe that the $D$-topology contains the product topology by definition of product diffeology. Let $A$ be a subset of $\prod_{\omega} \mathbb{R}$ such that it is not in the product topology. In other words, there exists $a=\left(a_{0}, a_{1}, \ldots\right) \in A$ such that no open neighborhood of 
$a$ in the product topology is contained in $A$. Let $\epsilon_{i} \in \mathbb{R}^{>0}$ for $i \in \mathbb{N}$, and let

$$
U_{i}=\prod_{j=0}^{i}\left(a_{j}-\epsilon_{i}, a_{j}+\epsilon_{i}\right) \times \prod_{k \in \mathbb{N} \backslash\{0,1, \ldots, i\}} \mathbb{R} .
$$

Clearly, $U_{i}$ is an open neighborhood of $a$ in the product topology, so there exists $b_{i}=\left(b_{i 0}, b_{i 1}, \ldots\right) \in U_{i} \backslash A$. We can choose $\epsilon_{i}$ 's so that $b_{i n} \rightarrow a_{n}$ fast as $i \rightarrow \infty$ for each $n \in \mathbb{N}$; for definition of fast convergence, see [KMl, page 17]. By the Special Curve Lemma [KM, page 18], there exists a smooth map $c: \mathbb{R} \rightarrow \prod_{\omega} \mathbb{R}$ such that $c(1 / n)=b_{n}$ and $c(0)=a$. Therefore, $A$ is not $D$-open.

Claim 3: There is no smooth linear map $q: \prod_{\omega} \mathbb{R} \rightarrow C^{\infty}(\mathbb{R}, \mathbb{R})$ such that $\phi \circ q=1 \prod_{\omega} \mathbb{R}$.

To prove this, it is enough to show that for every smooth linear map

$$
q: \prod_{\omega} \mathbb{R} \rightarrow C^{\infty}(\mathbb{R}, \mathbb{R}),
$$

$\phi \circ q$ is not injective. By [CSW, Corollary 4.10], the $D$-topology on $C^{\infty}(\mathbb{R}, \mathbb{R})$ coincides with the weak topology, or by [CSW, Proposition 4.2], the $D$-topology on $C^{\infty}(\mathbb{R}, \mathbb{R})$ contains the compact-open topology. Hence,

$$
A=\left\{f \in C^{\infty}(\mathbb{R}, \mathbb{R})|| f(t) \mid<1 \text { for all } t \text { with }|t| \leqslant 1\right\}
$$

is $D$-open in $C^{\infty}(\mathbb{R}, \mathbb{R})$, which implies that $q^{-1}(A)$ is $D$-open in $\prod_{\omega} \mathbb{R}$ by the smoothness of $q$. It is clear that $\overrightarrow{0}=(0,0, \ldots) \in q^{-1}(A)$ since the zero function is in $A$ and $q$ is linear. In Claim 2 we proved that the $D$-topology on $\prod_{\omega} \mathbb{R}$ coincides with the product topology, so there exists $N \in \mathbb{Z}^{+}$such that

$$
\overrightarrow{0} \in B=\left\{x \in \prod_{\omega} \mathbb{R} \mid x_{n}<1 / N \text { for all } n \leqslant N\right\} \subseteq q^{-1}(A) .
$$

Therefore, for any $x \in \prod_{\omega} \mathbb{R}$ with $x_{n}=0$ for all $n \leqslant N, k x \in B$ for every $k \in \mathbb{N}$. So, by the linearity of $q$,

$$
k(q(x))=q(k x) \in q(B) \subseteq A,
$$

which implies that $q(x)(t)=0$ for all $t$ with $|t| \leqslant 1$ by definition of $A$. Therefore,

$$
(\phi \circ q(x))_{n}=\frac{\partial^{n} q(x)}{\partial t^{n}}(0)=0 \text { for all } n \in \mathbb{N} ;
$$

that is, $\phi \circ q$ is not injective.

Conclusion: Combining Claims 1 and 3 with Theorem [1], we know that

$$
0 \longrightarrow K \longrightarrow C^{\infty}(\mathbb{R}, \mathbb{R}) \stackrel{\phi}{\longrightarrow} \prod_{\omega} \mathbb{R} \longrightarrow 0
$$

is a short exact sequence in $\mathfrak{D V e c t}$, but it does not split smoothly.

Remark 4.5 (Generalized Borel's lemma, the global version). Combining Claim 1 of the above example with Corollary $\mathbf{6 . 5}$, we can restate the generalized Borel's lemma as follows: For any open subset $U$ of a Euclidean space and any set of smooth functions $\left\{f_{i}: U \rightarrow \mathbb{R}\right\}_{i \in \mathbb{N}}$, there exists a smooth map $F: U \times \mathbb{R} \rightarrow \mathbb{R}$ such 
that $\frac{\partial^{k} F}{\partial y^{k}}(x, 0)=f_{k}(x)$ for all $k \in \mathbb{N}$ and $x \in U$. And this analytic statement is equivalent to the geometric statement that the map $\phi$ introduced in Example 6.3 is a linear subduction.

\section{Fine diffeological vector spaces}

In this section, we recall the definition and some basic properties of fine diffeological vector spaces. They behave like vector spaces, except for infinite products and duals. We also present some examples and non-examples of fine diffeological vector spaces. The analytic proof of Example relates to an interesting problem in analysis. We prove that the free diffeological vector space generated by a diffeological space $X$ is fine if and only if $X$ is discrete, and that every fine diffeological vector space is Frölicher.

Theorem 5.1. The forgetful functor $\mathfrak{D V e c t} \rightarrow \mathfrak{V}$ ect has both left and right adjoints.

Proof. The right adjoint is given by sending a vector space to the same space with the indiscrete diffeology, and the left adjoint is given by sending a vector space to the same space with the fine diffeology, which is the smallest diffeology on the vector

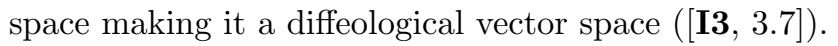

Definition $5.2([\mathbb{[ 3}, 3.7])$. A vector space with the fine diffeology is called a fine diffeological vector space.

Here are some basic properties of fine diffeological vector spaces.

1. Fine diffeology on a vector space $V$ is generated by all (injective) linear maps $\mathbb{R}^{n} \rightarrow V$ for all $n \in \mathbb{N}$; see [43, 3.8]. (In particular, this implies that the diffeological dimension of any diffeological vector space is always greater than or

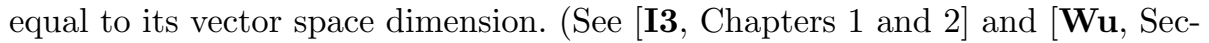
tion 1.8] for the definition and basic properties of the diffeological dimension of a diffeological space.) The equality does not always hold. For example, the diffeological dimension of $\mathbb{R}_{\text {ind }}$ is $\infty$, while its vector space dimension is 1.)

2. The fine diffeology on $\mathbb{R}^{n}$ is the standard diffeology; see $[\mathbf{L 3}$, Exercise 66 on page 71].

3. There is an equivalence between the category of fine diffeological vector spaces with smooth linear maps and the category $\mathfrak{V}$ ect of vector spaces with linear

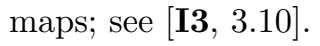

4. Every linear subspace of a fine diffeological vector space is again a fine diffeological vector space.

5. Finite product of fine diffeological vector spaces is again a fine diffeological vector space. But in general this is not true for infinite product; see Example

6. Every quotient vector space of a fine diffeological vector space is again a fine diffeological vector space. Any coproduct of fine diffeological vector spaces in $\mathfrak{D V e c t}$ is again a fine diffeological vector space, which follows from the description of the coproduct diffeology in $\mathfrak{D V e c t}$ (see the proof of Proposition 5.2 ) and (回). Therefore, every colimit of fine diffeological vector spaces in $\mathfrak{D V}$ Vect is again a fine diffeological vector space. 
7. If $V$ is a fine diffeological vector space and $W$ is a diffeological vector space, then $L^{\infty}(V, W)=L(V, W)$ by Theorem 5 . ; that is, a smooth linear map $V \rightarrow W$ is the same as a linear map $V \rightarrow W$. It follows that every fine diffeological vector space is a free diffeological vector space generated by any basis with the discrete diffeology. The converse is also true; see Theorem 3 .

8. Let $V$ be a fine diffeological vector space, and let $A$ be a basis of $V$. Then $V$ is isomorphic to the coproduct of $|A|$-copies of $\mathbb{R}$ in $\mathfrak{D}$ Vect.

Let $W$ be an arbitrary diffeological vector space. Then $L^{\infty}(V, W)$ is isomorphic in $\mathfrak{D V e c t}$ to the product of $|A|$-copies of $W$. In particular, if $V=\mathbb{R}^{n}$ and $W$ is fine, then $L^{\infty}(V, W)$ is also fine.

9. Tensor product of finitely many fine diffeological vector spaces is again a fine diffeological vector space. This follows from (प), Proposition $\mathbf{3 . J}$, and the fact that finite product of discrete diffeological spaces is again a discrete diffeological space.

10. Let $V$ be a fine diffeological vector space. Then the canonical map $V \rightarrow \tilde{D}^{2}(V)$ is injective. Furthermore, if $V$ is also finite dimensional, then $\tilde{D}(V)$ is fine and this canonical map is an isomorphism in $\mathfrak{D}$ Vect. But in general, $\tilde{D}(V)$ may not be fine; see Example 5.5 .

11. Let $0 \rightarrow V_{1} \rightarrow V_{2} \rightarrow V_{3} \rightarrow 0$ be a short exact sequence of diffeological vector spaces. If $V_{3}$ is fine, then this short exact sequence splits smoothly by (U) and Theorem [.]. If $V_{2}$ is fine, then this short exact sequence splits smoothly as well by (प) and Theorem [.]त, and in particular, we conclude that both $V_{1}$ and $V_{3}$ are fine by $(\mathbb{G})$ and $(\mathbf{G})$.

As an immediate consequence, it is not true that for every diffeological vector space $V$, there is a linear subduction from a fine diffeological vector space to $V$.

Theorem 5.3. Let $X$ be a diffeological space such that $F(X)$ is a fine diffeological vector space. Then $X$ is discrete.

Proof. Assume that there exist a plot $p: U \rightarrow X$ and a point $u_{0} \in U$ such that for every open neighborhood $V$ of $u_{0}$ in $U$, there exists $v \in V$ such that $p(v) \neq p\left(u_{0}\right)$. Consider the plot $q: \mathbb{R} \times U \rightarrow F(X)$ given by $(t, u) \mapsto t[p(u)]$. Since $F(X)$ is fine, $\left.q\right|_{A \times B}=l \circ f$ for some $n \in \mathbb{N}$, some open neighborhoods $A$ of $t_{0} \neq 0$ in $\mathbb{R}$ and $B$ of $u_{0}$ in $U$, a smooth function $f: A \times B \rightarrow \mathbb{R}^{n}$, and a linear map $l: \mathbb{R}^{n} \rightarrow F(X)$. Without loss of generality, we may assume that $l$ sends the canonical basis of $\mathbb{R}^{n}$ to the canonical basis of $F(X)$; that is, for every $i \in\{1,2 \ldots, n\}, l\left(e_{i}\right)=\left[x_{i}\right]$ for some $x_{i} \in X$. Then the equality $\left.q\right|_{A \times B}=l \circ f$ simply implies that $f$ cannot be smooth. Therefore, $X$ is a discrete diffeological space.

Example 5.4. The countable product $\prod_{\omega} \mathbb{R}$ of fine diffeological vector spaces is not fine. The reason is, if $\prod_{\omega} \mathbb{R}$ were fine, then by Property $(\mathbb{\square})$, the short exact sequence of diffeological vector spaces in Example 4.3 would split smoothly.

Here is another way to see this fact. Let $\lambda$ be a fixed smooth bump function; that is, $\lambda \in C^{\infty}(\mathbb{R}, \mathbb{R})$ such that $\operatorname{supp}(\lambda) \subset(0,1)$ and $\operatorname{Im}(\lambda)=[0,1]$. Let $\Phi: \mathbb{R} \rightarrow \prod_{\omega} \mathbb{R}$ be defined by

$$
(\Phi(t))_{n}=\lambda\left[n(n+1)\left(t-\frac{1}{n+1}\right)\right] .
$$

Then $\Phi$ is smooth, and the image of any neighborhood of $0 \in \mathbb{R}$ under $\Phi$ does not 
live in any finite dimensional linear subspace of $\prod_{\omega} \mathbb{R}$. Therefore, $\prod_{\omega} \mathbb{R}$ is not fine.

Furthermore, $\oplus_{\omega} \mathbb{R}$ is a linear subspace of $\prod_{\omega} \mathbb{R}$. But by using the function $\Phi$, it is easy to see that the sub-diffeology from $\prod_{\omega} \mathbb{R}$ is different from the fine diffeology on $\oplus_{\omega} \mathbb{R}$.

Let $i: \mathbb{R} \rightarrow \prod_{\omega} \mathbb{R}$ be defined by $x \mapsto(x, 0,0, \ldots)$. One can check easily that $i$ is a linear induction such that $p_{0} \circ i=1_{\mathbb{R}}$. In other words, although $\prod_{\omega} \mathbb{R}$ is not a fine diffeological vector space, it has a smooth direct summand of a fine diffeological vector space.

Example 5.5. The dual of a fine diffeological vector space may not be fine. For example, if $V=\oplus_{\omega} \mathbb{R}$, the coproduct of countably many copies of $\mathbb{R}$ in $\mathfrak{D V e c t}$, then $\tilde{D}(V)=\prod_{\omega} \mathbb{R}$, the product of countably many copies of $\mathbb{R}$ in $\mathfrak{D}$ Vect. By Example $\square$, we know that $\tilde{D}(V)$ is not fine.

Example 5.6. The diffeological vector space $C^{\infty}(\mathbb{R}, \mathbb{R})$ is not fine.

Here is an analytic proof. Assume that the diffeological vector space $C^{\infty}(\mathbb{R}, \mathbb{R})$ is fine. Let $f: \mathbb{R} \rightarrow C^{\infty}(\mathbb{R}, \mathbb{R})$ be the plot with $f(x)(y)=e^{x y}$. Then there exist an open neighborhood $U$ of 0 in $\mathbb{R}$, an integer $n \in \mathbb{N}$, a smooth map $g: U \rightarrow \mathbb{R}^{n}$, and a linear $\operatorname{map} h: \mathbb{R}^{n} \rightarrow C^{\infty}(\mathbb{R}, \mathbb{R})$ such that $\left.f\right|_{U}=h \circ g$. In other words, for any $(x, y) \in U \times \mathbb{R}$, $e^{x y}=\sum_{k=1}^{n} g_{k}(x) h_{k}(y)$ for some smooth functions $g_{1}, \ldots, g_{n}, h_{1}, \ldots, h_{n} \in C^{\infty}(\mathbb{R}, \mathbb{R})$.

Now fix $\delta \in \mathbb{R}^{>0}$ such that $\delta, 2 \delta, \ldots, n \delta \in U$. The $(n+1) \times(n+1)$ matrix $A:=$ $\left[e^{i j \delta^{2}}\right]_{i, j=0}^{n}$ is equal to

$$
\sum_{k=1}^{n}\left[g_{k}(0), g_{k}(\delta), \ldots, g_{k}(n \delta)\right]^{T}\left[h_{k}(0), h_{k}(\delta), \ldots, h_{k}(n \delta)\right],
$$

i.e., the sum of $n$ rank- 1 matrices, hence singular. On the other hand, $A$ is a Vandermonde matrix, so its determinant is

$$
\prod_{\substack{i, j=0, \ldots, n \\ i>j}}\left(e^{i \delta^{2}}-e^{j \delta^{2}}\right) \neq 0 .
$$

The contradiction implies that the diffeological vector space $C^{\infty}(\mathbb{R}, \mathbb{R})$ is not fine.

Here is an algebraic proof. In Claim 1 of Example 4.3, we have shown that

$$
0 \longrightarrow K \longrightarrow C^{\infty}(\mathbb{R}, \mathbb{R}) \stackrel{\phi}{\longrightarrow} \prod_{\omega} \mathbb{R} \longrightarrow 0
$$

is a short exact sequence of diffeological vector spaces. Now we have two ways to argue that $C^{\infty}(\mathbb{R}, \mathbb{R})$ is not fine. The first way is, if $C^{\infty}(\mathbb{R}, \mathbb{R})$ is fine, then so is $\prod_{\omega} \mathbb{R}$ by Property $(\mathbb{U})$, but it is not by Example $\square$. The second way is, if $C^{\infty}(\mathbb{R}, \mathbb{R})$ is

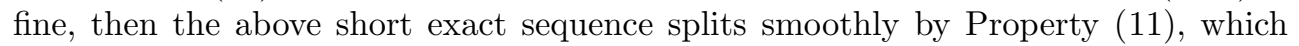
contradicts the conclusion of Example 4.3].

Frölicher spaces are another well-studied generalization of smooth manifolds. We refer the reader to $[\mathbf{S t}]$ for definition of the category $\mathfrak{F}$ r of Frölicher spaces and smooth maps. There is an adjoint pair $F: \operatorname{Diff} \rightleftharpoons \mathfrak{F r}: G$; see [St]. We say that a diffeological space $X$ is Frölicher if there exists a Frölicher space $Y$ such that $G(Y)=X$.

Proposition 5.7. Every fine diffeological vector space is Frölicher. 
Proof. Let $V$ be a fine diffeological vector space. Write $\mathcal{F}=C^{\infty}(V, \mathbb{R})$ and write $\mathcal{C}=\left\{c: \mathbb{R} \rightarrow V \mid l \circ c \in C^{\infty}(\mathbb{R}, \mathbb{R})\right.$ for all $\left.l \in \mathcal{F}\right\}$. Then $(\mathcal{C}, V, \mathcal{F})$ is a Frölicher space.

We are left to show that for any open subset $U$ of $\mathbb{R}^{n}$, for any set map $f: U \rightarrow V$, if $l \circ f \in C^{\infty}(U, \mathbb{R})$ for every $l \in \mathcal{F}$, then $f$ is a plot of $V$.

Note that if $A$ is a basis of $V$, then $V$ is isomorphic to $\oplus_{a \in A} \mathbb{R}$ in $\mathfrak{D V e c t}$. Hence, a linear projection of $V$ to any 1-dimensional linear subspace is in $\mathcal{F}$. Therefore, it is enough to show that for any $u \in U$, there exists an open neighborhood $U^{\prime}$ of $u$, such that $\operatorname{Im}\left(\left.f\right|_{U^{\prime}}\right)$ is in a finite dimensional linear subspace of $V$.

Now assume that for every open neighborhood $U^{\prime}$ of $u$ in $U, \operatorname{Im}\left(\left.f\right|_{U^{\prime}}\right)$ is not in a finite dimensional linear subspace of $V$. Then there exists a sequence $u_{n} \rightarrow u$ in $U$ such that

$$
\left\{f\left(u_{n}\right)\right\}_{n \in \mathbb{N}} \text { is a linearly independent subset of } V \text {, if } f(u)=0 \text {; }
$$

or $\left\{f\left(u_{n}\right)\right\}_{n \in \mathbb{N}} \cup\{f(u)\}$ is a linearly independent subset of $V$, if $f(u) \neq 0$.

We can extend this linearly independent subset to a basis $B$ of $V$. Then by the universal property of coproduct in $\mathfrak{D V e c t}, g: V \rightarrow \mathbb{R}$ defined by the linear extension of the map

$$
\left\{\begin{array}{l}
f\left(u_{n}\right) \mapsto 1 \text { for every } n \in \mathbb{N} \\
\text { other elements in } B \mapsto 0
\end{array}\right.
$$

is in $\mathcal{F}$. But clearly $g \circ f$ is not continuous, hence not smooth, contracting the assumption that $l \circ f \in C^{\infty}(U, \mathbb{R})$ for every $l \in \mathcal{F}$.

\section{Projective diffeological vector spaces}

In this section, we introduce a large class of diffeological vector spaces called projective diffeological vector spaces. They have the lifting property with respect to all linear subductions. Fine diffeological vector spaces and the free diffeological vector spaces generated by smooth manifolds are such examples. We give several equivalent characterizations of projective diffeological vector spaces in this section and next. However, not every (free) diffeological vector space is projective. But there are enough projectives in the category $\mathfrak{D V e c t}$ of diffeological vector spaces.

Definition 6.1. We call a diffeological vector space $V$ projective, if for every linear subduction $f: W_{1} \rightarrow W_{2}$ and every smooth linear map $g: V \rightarrow W_{2}$, there exists a smooth linear map $h: V \rightarrow W_{1}$ such that $g=f \circ h$.

Proposition 6.2. Let $X$ be a diffeological space. Then the free diffeological vector space $F(X)$ generated by $X$ is projective if and only if for any linear subduction $f: W_{1} \rightarrow W_{2}$ and any smooth map $g: X \rightarrow W_{2}$, there exists a smooth map $h: X \rightarrow$ $W_{1}$ making the following diagram commutative:

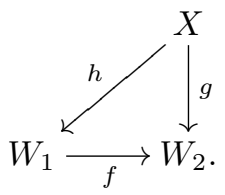


Proof. This follows directly from definition of projective diffeological vector space and the universal property of free diffeological vector space generated by a diffeological space.

Corollary 6.3. Every fine diffeological vector space is projective.

Proof. This follows directly from Proposition $\mathbf{5}$ together with Property (प) that every fine diffeological vector space is a free diffeological vector space generated by a discrete diffeological space.

Corollary 6.4. The free diffeological vector space generated by a smooth manifold is projective.

Proof. Let $M$ be a smooth manifold. For any linear subduction $f: W_{1} \rightarrow W_{2}$ and any smooth map $g: M \rightarrow W_{2}$, by Proposition [.2, we only need to construct a smooth map $h: M \rightarrow W_{1}$ such that $g=f \circ h$.

Since $f: W_{1} \rightarrow W_{2}$ is a subduction and $M$ is a smooth manifold, we can find an atlas $\left\{U_{i}\right\}_{i \in I}$ of $M$ such that for each $i$, there exists a smooth map $h_{i}: U_{i} \rightarrow W_{1}$ making the following diagram commutative:

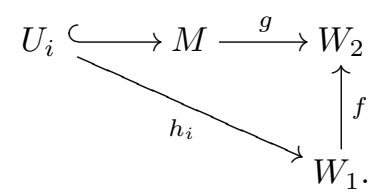

Let $\left\{\rho_{i}\right\}_{i \in I}$ be a smooth partition of unity subordinate to this covering $\left\{U_{i}\right\}_{i \in I}$ of $M$. Since $W_{1}$ is a diffeological vector space, the map $h: M \rightarrow W_{1}$ defined by $h(m)=\sum_{i \in I} \rho_{i}(m) h_{i}(m)$ is smooth. It is easy to check that $f(h(m))=g(m)$ for all $m \in M$.

By Theorem 2.3, it is clear that Corollary $\mathbf{6 . 4}$ provides a lot of projective but not fine diffeological vector spaces. Moreover, we have the following immediate consequence from the proof of Corollary 6.4, which will be essential for homological algebra of diffeological vector spaces:

Corollary 6.5 (Global Lifting). For any linear subduction $W_{1} \rightarrow W_{2}$, every plot of $W_{2}$ globally lifts to a plot of $W_{1}$.

Remark 6.6. By [13], 8.15], we know that every linear subduction $\pi: W_{1} \rightarrow W_{2}$ is a diffeological principal bundle; see [103, Chapter 8]. By Corollary [5.5, the pullback in $\mathfrak{D i f f}$ along any plot $p: U \rightarrow W_{2}$ is globally trivial. So for any $(u, w) \in U \times W_{1}$ with $p(u)=\pi(w)$, there exists a smooth map $f: U \rightarrow W_{1}$ such that $p=\pi \circ f$ and $f(u)=w$.

Example 6.7.

1. Let

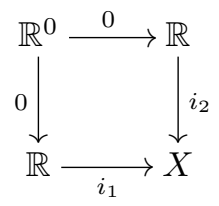

be a pushout diagram in $\mathfrak{D}$ iff. Then $F(X)$ is projective. 
Here is the proof. Let $f: W_{1} \rightarrow W_{2}$ be a linear subduction, and let $g: X \rightarrow W_{2}$ be a smooth map. By Corollary $\overline{6.4}$, there exist smooth maps $\alpha, \bar{\beta}: \mathbb{R} \rightarrow W_{1}$ such that $f \circ \alpha=g \circ i_{1}$ and $f \circ \bar{\beta}=g \circ i_{2}$. Since $W_{1}$ is a diffeological vector space, we can define $\beta: \mathbb{R} \rightarrow W_{1}$ by $\beta(y)=\bar{\beta}(y)-\bar{\beta}(0)+\alpha(0)$. Then the map $\beta$ is smooth, and the linearity of $f$ implies that $f \circ \beta=g \circ i_{2}$. Now $\alpha(0)=\beta(0)$. So we have a smooth map $h: X \rightarrow W_{1}$ such that $f \circ h=g$. The result then follows from Proposition [.2.

2. Let $X$ be the union of the coordinate axes in $\mathbb{R}^{2}$ with the sub-diffeology. Then $F(X)$ is projective.

Here is the proof. Write $i_{1}, i_{2}: \mathbb{R} \rightarrow X$ for the smooth maps defined by $i_{1}(x)=$ $(x, 0)$ and $i_{2}(y)=(0, y)$, and write $i: X \rightarrow \mathbb{R}^{2}$ for the inclusion. Let $f: W_{1} \rightarrow W_{2}$ be a linear subduction, and let $g: X \rightarrow W_{2}$ be a smooth map. Since $W_{2}$ is a diffeological vector space, we can define $G: \mathbb{R}^{2} \rightarrow W_{2}$ by $G(x, y)=g\left(i_{1}(x)\right)+$ $g\left(i_{2}(y)\right)-g\left(i_{1}(0)\right)$. Then $G$ is a smooth map, and $G \circ i=g$. By Corollary [.4, there exists a smooth map $H: \mathbb{R}^{2} \rightarrow W_{1}$ such that $f \circ H=G$. Hence, $h:=$ $H \circ i: X \rightarrow W_{1}$ is a smooth map such that $f \circ h=g$.

Remark 6.8. More generally, using the terminology in [CWT], we have the following: Let $X$ and $Y$ be diffeological spaces. If either $X$ is cofibrant, or $F(Y)$ is a projective diffeological vector space and there is a cofibration $X \rightarrow Y$, then $F(X)$ is a projective diffeological vector space. This follows from the fact that every linear subduction is a trivial fibration.

However, not every (free) diffeological vector space is projective:

\section{Example 6.9.}

1. Not every diffeological vector space is projective. From Example 4.3, we know that $\prod_{\omega} \mathbb{R}$ is such an example.

2. More surprisingly, not every free diffeological vector space is projective.

Let $\alpha$ be an irrational number, and let $T_{\alpha}$ be the quotient group $\mathbb{R} /(\mathbb{Z}+\alpha \mathbb{Z})$ with the quotient diffeology. We call $T_{\alpha}$ the 1-dimensional irrational torus of slope $\boldsymbol{\alpha}$. Write $\pi: \mathbb{R} \rightarrow T_{\alpha}$ for the quotient map. Then $F(\pi): F(\mathbb{R}) \rightarrow F\left(T_{\alpha}\right)$ is a linear subduction. We claim that $F\left(T_{\alpha}\right)$ is not projective.

By Proposition [.2, we only need to show that there exists no smooth map $h: T_{\alpha} \rightarrow F(\mathbb{R})$ such that $i_{T_{\alpha}}=F(\pi) \circ h$, where $i_{T_{\alpha}}: T_{\alpha} \rightarrow F\left(T_{\alpha}\right)$ is the canonical smooth map. Otherwise, $f:=h \circ \pi \in C^{\infty}(\mathbb{R}, F(\mathbb{R}))$ and $F(\pi)(f(x))=i_{T_{\alpha}}(\pi(x))$ for all $x \in \mathbb{R}$. On the one hand, $f \in C^{\infty}(\mathbb{R}, F(\mathbb{R}))$ implies that there exists a connected open neighborhood $A$ of 0 in $\mathbb{R}$ together with smooth maps $\beta_{i}: A \rightarrow \mathbb{R}$ (viewed as scalars) and $\gamma_{i}: A \rightarrow \mathbb{R}$ (viewed as base) with $1 \leqslant i \leqslant n$ for some minimum $n \in \mathbb{Z}^{+}$, such that $f(x)=\sum_{i=1}^{n} \beta_{i}(x)\left[\gamma_{i}(x)\right]$ for all $x \in A$. Since $(\mathbb{Z}+\alpha \mathbb{Z}) \cap A$ is dense in $A$, the map $\gamma_{i}$ must be constant for each $i$. On the other hand, since $\left.\pi\right|_{A}: A \rightarrow T_{\alpha}$ is surjective, $F(\pi)(f(x))=i_{T_{\alpha}}(\pi(x))$ for all $x \in A$ implies that not every $\gamma_{i}$ is constant. So we reach a contradiction.

Here are some immediate consequences of this example:

- Together with Remark $\mathbf{6 . 8}$, we get an independent proof that every 1dimensional irrational torus $T_{\alpha}$ is non-cofibrant (see [CWT], Example 4.27] for an alternative proof). 
- The diffeological vector space $F\left(T_{\alpha}\right)$ is not fine.

By a similar argument, one can show that $F^{2}\left(T_{\alpha}\right):=F\left(F\left(T_{\alpha}\right)\right)$, the free diffeological vector space generated by $F\left(T_{\alpha}\right)$, is also not projective. More generally, none of $F^{n}\left(T_{\alpha}\right):=F\left(F^{n-1}\left(T_{\alpha}\right)\right)$ is projective for $n \in \mathbb{Z}^{+}$.

3. $\mathbb{R}_{\text {ind }}$ is not projective, since there is no smooth linear map $h: \mathbb{R}_{\text {ind }} \rightarrow F\left(\mathbb{R}_{\text {ind }}\right)$ such that $\eta \circ h=1_{\mathbb{R}_{\text {ind }}}$, where $\eta: F\left(\mathbb{R}_{\text {ind }}\right) \rightarrow \mathbb{R}_{\text {ind }}$ is the canonical smooth linear map induced by $1_{\mathbb{R}_{\text {ind }}}: \mathbb{R}_{\text {ind }} \rightarrow \mathbb{R}_{\text {ind }}$.

Here is another consequence of Example 6 प्ञ(2):

Lemma 6.10. Projective diffeological vector spaces are not preserved under arbitrary colimits in $\mathfrak{D}$ Vect.

Proof. By [CSW, Proposition 2.7], every diffeological space $X$ is a colimit of open subsets $U$ of Euclidean spaces, i.e., $X=\operatorname{colim} U$. The free functor $F: \mathfrak{D i f f} \rightarrow \mathfrak{D V}$ Vect is a left adjoint (Proposition $[.5$ ), so it commutes with colimits, i.e., $F(X)=\operatorname{colim} F(U)$.

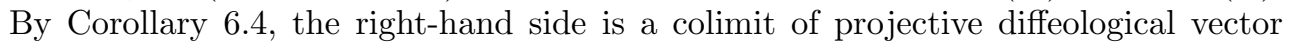
spaces, while by Example $\mathbf{E . g}(2)$, we know that the left-hand side can fail to be a projective diffeological vector space.

Here are some basic properties for projective diffeological vector spaces:

\section{Proposition 6.11.}

1. A diffeological vector space $V$ is projective if and only if every linear subduction $W \rightarrow V$ splits smoothly.

2. Let $\left\{V_{i}\right\}_{i \in I}$ be a set of diffeological vector spaces. Then each $V_{i}$ is projective if and only if the coproduct $\oplus_{i \in I} V_{i}$ in $\mathfrak{D}$ Vect is projective.

3. Projective diffeological vector spaces are closed under retracts in $\mathfrak{D}$ Vect. That is, if $f: V \rightarrow W$ and $g: W \rightarrow V$ are smooth linear maps between diffeological vector spaces such that $g \circ f=1_{V}$ and $W$ is projective, then $V$ is also projective.

4. Let $0 \rightarrow V_{1} \rightarrow V_{2} \rightarrow V_{3} \rightarrow 0$ be a short exact sequence of diffeological vector spaces. If $V_{3}$ is projective, then this short exact sequence splits smoothly. In particular, every projective diffeological vector space is a smooth direct summand of a free diffeological vector space, but the converse is not true in general.

Proof. (1) follows from the definition of projective diffeological vector space and the fact that linear subductions are closed under pullbacks in $\mathfrak{D}$ Vect. The rest are formal.

\section{Example 6.12.}

1. Let $C_{n}$ be the cyclic subgroup of order $n$ of the multiplicative group $S^{1}$. Let $C_{n}$ act on $\mathbb{R}^{2}$ by rotation, and write $X$ for the quotient diffeological space with the quotient map $\pi: \mathbb{R}^{2} \rightarrow X$. Then $F(X)$ is a projective diffeological vector space. Here is the proof. Define $f: X \rightarrow F\left(\mathbb{R}^{2}\right)$ by $x \mapsto \sum \frac{1}{n}[\bar{x}]$, where the sum is over all $\bar{x} \in \pi^{-1}(x)$. Clearly $f$ is a smooth map such that $F(X)$ is a retract in $\mathfrak{D V e c t}$ of the projective diffeological vector space $F\left(\mathbb{R}^{2}\right)$. Hence, $F(X)$ is also projective by Proposition $\mathbf{E}$. 
2. Similarly, if $X_{n}$ is the quotient diffeological space $\mathbb{R}^{n} /\{ \pm 1\}$, then $F\left(X_{n}\right)$ is a projective diffeological vector space.

Recall from Example $\mathbf{E}(2)$ that the domain of the canonical linear subduction $F^{2}\left(T_{\alpha}\right) \rightarrow F\left(T_{\alpha}\right)$ is not projective. So the proof of Corollary [.].3 does not provide us a functorial way to find a projective diffeological vector space $V$ together with a linear subduction $V \rightarrow F\left(T_{\alpha}\right)$. However, there is a linear subduction $F(\pi): F(\mathbb{R}) \rightarrow F\left(T_{\alpha}\right)$ whose domain is a projective diffeological vector space.

Theorem 6.13. The category $\mathfrak{D V e c t ~ h a s ~ e n o u g h ~ p r o j e c t i v e s . ~ T h a t ~ i s , ~ f o r ~ a n y ~ d i f f e o - ~}$ logical vector space $V$, there exists a projective diffeological vector space $P(V)$ together with a linear subduction $P(V) \rightarrow V$.

Proof. Let $V$ be an arbitrary diffeological vector space. We construct $P(V)$ as the coproduct in $\mathfrak{D}$ Vect of all $F(U)$ indexed by all plots $U \rightarrow V$. By the universal property of free diffeological vector space generated by a diffeological space, there is a canonical smooth linear map $F(U) \rightarrow V$. Therefore, by the universal property of coproduct in $\mathfrak{D V e c t}$, there is a smooth linear map $P(V) \rightarrow V$. By construction, it is easy to see

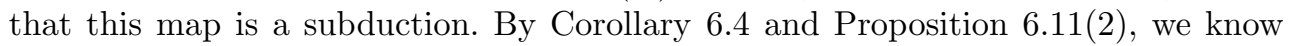
that $P(V)$ is a projective diffeological vector space.

Note that since the free functor $F: \mathfrak{D i f f} \rightarrow \mathfrak{D V e c t}$ is a left adjoint (Proposition $\mathbf{B .}$ ), $P(V)$ constructed in the proof of the above theorem is actually a free diffeological vector space.

Of course, given a diffeological vector space $V$, the projective diffeological vector space $P(V)$ constructed in the proof of the above theorem is functorial but huge. There is a natural transformation $P \rightarrow 1$, and $f: V \rightarrow W$ is a linear subduction if and only if $P(f): P(V) \rightarrow P(W)$ is. Here is a non-functorial but much smaller construction, whose proof is similar to the proof of the above theorem:

Proposition 6.14. Let $V$ be a diffeological vector space, and let $\left\{f_{i}: U_{i} \rightarrow V\right\}$ be a generating set of the diffeology on $V$. Then $\sum \eta_{i}: \oplus F\left(U_{i}\right) \rightarrow V$ is a linear subduction with the domain a projective diffeological vector space. Here $\eta_{i}$ is the smooth linear map induced by $f_{i}$ for each $i$.

Here is an immediate consequence:

Corollary 6.15. Every projective diffeological vector space is a smooth direct summand of a coproduct of free diffeological vector spaces generated by Euclidean spaces.

Proposition 6.16. A diffeological vector space $V$ is projective if and only if the functor $L^{\infty}(V,-): \mathfrak{D V e c t} \rightarrow \mathfrak{D V}$ ect preserves short exact sequences.

Proof. $(\Leftarrow)$ This is clear.

$(\Rightarrow)$ Since $\mathfrak{D V e c t}$ has enough projectives, $V$ is a retract in $\mathfrak{D}$ Vect of $P(V)$, where $P(V)$ was introduced in the proof of Theorem [.]3. Since the functor $L^{\infty}(W,-)$ is a right adjoint for any diffeological vector space $W$ (Theorem $\mathbf{3 . 8}$ ) and subductions are closed under retracts, we are left to show that $L^{\infty}(P(V),-)$ preserves subductions. By Remark $\mathbf{5 . 6}$ and Corollary $\mathbf{5 . 5}$, we are left to show that for any open subset $U$ of a Euclidean space, the functor $C^{\infty}(U,-): \mathfrak{D V}$ ect $\rightarrow \mathfrak{D V e c t}$ preserves linear subductions. This then follows from Corollary [..4. 
As an easy corollary, we have:

Corollary 6.17. If $V$ and $W$ are projective diffeological vector spaces, then so is $V \otimes W$.

\section{Homological algebra for diffeological vector spaces}

In this section, we develop (relative) homological algebra for diffeological vector spaces based on the results we get in previous sections about linear subductions and projective diffeological vector spaces. We show that every diffeological vector space has a diffeological projective resolution, which is unique up to diffeological chain homotopy equivalence. Shanuel's lemma, the Horseshoe Lemma, and the (Short) Five Lemma still hold in this setting. From a short exact sequence of diffeological (co)chain complexes, we get a long sequence in $\mathfrak{D V e c t}$ which is exact in $\mathfrak{V}$ ect. Then we define diffeological vector spaces $\operatorname{Ext}^{n}(V, W)$ for any diffeological vector spaces $V, W$ and any $n \in \mathbb{N}$. For any short exact sequence of diffeological vector spaces in the first or second variable, we get a long sequence of such diffeological vector spaces which is exact in $\mathfrak{V}$ ect. Finally, we show that $\operatorname{Ext}^{1}(V, W)$ classifies all short exact sequences in $\mathfrak{D V e c t}$ of the form $0 \rightarrow W \rightarrow A \rightarrow V \rightarrow 0$ up to equivalence.

As usual, we define the category $\mathfrak{D C h}$ of diffeological chain complexes to be the full subcategory of the functor category $\mathfrak{D V e c t}{ }^{\mathbb{Z}}$ consisting of objects in which the composition of every two consecutive arrows is 0 , where $\mathbb{Z}$ is viewed as a poset of integers with the opposite ordering. The morphisms in $\mathfrak{D C h}$ are called diffeological chain maps. For any $n \in \mathbb{Z}$, there exists a functor $H_{n}: \mathfrak{D C h} \rightarrow \mathfrak{D V}$ Vect defined by $H_{n}(\boldsymbol{V})=\operatorname{ker}\left(d_{n}\right) / \operatorname{Im}\left(d_{n+1}\right)$, where

$$
V_{n+1} \stackrel{d_{n+1}}{\longrightarrow} V_{n} \stackrel{d_{n}}{\longrightarrow} V_{n-1}
$$

is a piece in the diffeological chain complex $\mathbf{V}$, both $\operatorname{ker}\left(d_{n}\right)$ and $\operatorname{Im}\left(d_{n+1}\right)$ are equipped with the sub-diffeologies of $V_{n}$, and $H_{n}(\mathbf{V})$ is equipped with the quotient diffeology. We call $H_{n}(\mathbf{V})$ the $\boldsymbol{n}^{\boldsymbol{t h}}$ homology of $\mathbf{V}$. Two diffeological chain maps $\boldsymbol{f}, \boldsymbol{g}: \boldsymbol{V} \rightarrow \boldsymbol{W}$ are called diffeologically chain homotopic if there are smooth linear maps $h_{n}: V_{n} \rightarrow W_{n+1}$ for all $n \in \mathbb{Z}$ such that $f_{n}-g_{n}=h_{n-1} \circ d_{n}^{\boldsymbol{V}}+d_{n+1}^{\boldsymbol{W}} \circ h_{n}$ for each $n \in \mathbb{Z}$. This gives an equivalence relation on $\mathfrak{D C h}(\boldsymbol{V}, \boldsymbol{W})$, called the diffeological chain homotopy equivalence, which is compatible with compositions in $\mathfrak{D C h}$. Write $\mathfrak{h D C h}$ for the quotient category. Then the homology functors $H_{n}$ factor through the projection $\mathfrak{D C h} \rightarrow \mathfrak{h D C h}$. A diffeological chain map $\mathbf{f}: \mathbf{V} \rightarrow \mathbf{W}$ is called a diffeological homology isomorphism if for each $n \in \mathbb{Z}, H_{n}(\mathbf{f})$ is an isomorphism in $\mathfrak{D V e c t}$. A diffeological chain complex $\boldsymbol{V}$ is called diffeologically exact at the $\boldsymbol{n}^{\text {th }}$ spot if the induced map $0 \rightarrow V_{n+1} / \operatorname{ker}\left(d_{n+1}\right) \rightarrow V_{n} \rightarrow \operatorname{Im}\left(d_{n}\right) \rightarrow 0$ with $V_{n+1} / \operatorname{ker}\left(d_{n+1}\right)$ equipped with the quotient diffeology of $V_{n+1}$ and $\operatorname{Im}\left(d_{n}\right)$ equipped with the sub-diffeology of $V_{n-1}$ is a short exact sequence in $\mathfrak{D}$ Vect. A diffeological chain complex is called diffeologically exact if it is diffeologically exact at every spot. A diffeological projective resolution of a diffeological vector space $V$ is a diffeologically exact diffeological chain complex $\mathbf{V}$ such that $V_{-1}=V, V_{n}=0$ for every $n<-1$, and $V_{n}$ is projective for every $n \geqslant 0$. In this case, we write $\boldsymbol{P}(V)$ for the diffeological chain complex with $\boldsymbol{P}(V)_{n}=V_{n}$ for each $n \geqslant 0$ and $\boldsymbol{P}(V)_{n}=0$ for each 
$n<0$. Then the diffeological chain map from $\boldsymbol{P}(V)$ to the diffeological chain complex with $V$ concentrated at the $0^{\text {th }}$ spot is a diffeological homology isomorphism. By abuse of notation, we also call this diffeological chain map a diffeological projective resolution, and denote it by $\boldsymbol{P}(V) \rightarrow V$.

Remark 7.1. Note that if a diffeological chain complex $\boldsymbol{V}$ is diffeologically exact at the $n^{\text {th }}$ spot, then $H_{n}(\boldsymbol{V})=0$. But the converse is not true. For example, let $O(n)$ naturally act on $\mathbb{R}^{n}$, and let $X_{n}$ be the quotient space, for $n \in \mathbb{Z}^{+}$. Then $\mathbb{R}^{n} \rightarrow \mathbb{R}^{n+1}$ defined by $x \rightarrow(x, 0)$ induces a smooth map $i_{n}: X_{n} \rightarrow X_{n+1}$, and hence a smooth linear map $F\left(i_{n}\right): F\left(X_{n}\right) \rightarrow F\left(X_{n+1}\right)$. It is known in [12] that $i_{n}$ is a bijection, but not a diffeomorphism. So $F\left(i_{n}\right)$ is a bijection, but not an isomorphism in $\mathfrak{D}$ Vect. Let $\boldsymbol{V}$ be the diffeological chain complex with the only non-trivial diffeological vector spaces $F\left(X_{n}\right)$ and $F\left(X_{n+1}\right)$ at the $n^{t h}$ and $(n-1)^{t h}$ spots, and $d_{n}=F\left(i_{n}\right)$. Then $H_{i}(\boldsymbol{V})=0$ for every $i \in \mathbb{Z}$, but $\boldsymbol{V}$ is not diffeologically exact at both the $n^{\text {th }}$ and $(n-1)^{t h}$ spots.

Similarly, one can define the category $\mathfrak{D C h}{ }^{o}$ of diffeological cochain complexes and cohomology functors $H^{n}: \mathfrak{D C h}^{o} \rightarrow \mathfrak{D V}$ ect.

As the proof of [We, Lemma 2.2.5], one can show by inductively applying Theorem 6.3 that there is a diffeological projective resolution for every diffeological vector space. As the proof of [W], Comparison Theorem 2.2.6], for any diffeological projective resolution $\boldsymbol{V}$ of $V$ (or write as $\boldsymbol{P}(V) \rightarrow V$ ), any diffeologically exact diffeological chain complex $\boldsymbol{W}$ with $W_{i}=0$ for all $i \leqslant-2$, and any smooth linear map $f: V \rightarrow W_{-1}$, there exists a diffeological chain map $\boldsymbol{V} \rightarrow \boldsymbol{W}$ extending $f$, and the corresponding diffeological chain map $\boldsymbol{P}(V) \rightarrow \overline{\boldsymbol{W}}$ is unique up to diffeological chain homotopy equivalence, where $\overline{\boldsymbol{W}}$ is derived from $\boldsymbol{W}$ by replacing $W_{-1}$ by 0 . In particular, for any two diffeological projective resolutions $\boldsymbol{V}$ and $\boldsymbol{V}^{\prime}$ of $V$, there is an isomorphism between $\boldsymbol{P}(V)$ and $\boldsymbol{P}^{\prime}(V)$ in $\mathfrak{h D C h}$ from the corresponding diffeological chain maps between $\boldsymbol{V}$ and $\boldsymbol{V}^{\prime}$ extending $1_{V}: V \rightarrow V$.

Lemma 7.2 (Schanuel). Given short exact sequences of diffeological vector spaces

$$
0 \longrightarrow K \stackrel{i}{\longrightarrow} P \stackrel{\pi}{\longrightarrow} M \longrightarrow 0
$$

and

$$
0 \longrightarrow K^{\prime} \stackrel{i^{\prime}}{\longrightarrow} P^{\prime} \stackrel{\pi^{\prime}}{\longrightarrow} M \longrightarrow 0
$$

with both $P$ and $P^{\prime}$ projective, there is an isomorphism $K \oplus P^{\prime} \cong K^{\prime} \oplus P$ in $\mathfrak{D}$ Vect.

Proof. By assumption, we have the following commutative diagrams in $\mathfrak{D V e c t :}$

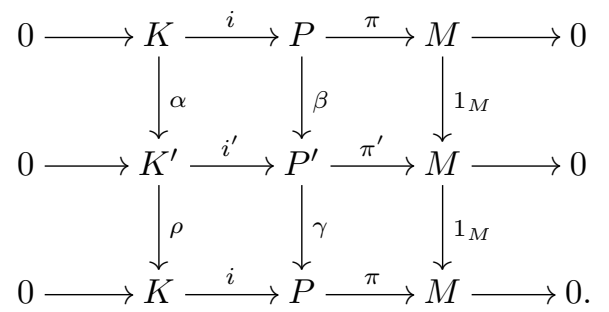


It is shown in the proof of $[\mathbb{R}$, Proposition 3.12] that

$$
0 \longrightarrow K \stackrel{(i, \alpha)}{\longrightarrow} P \oplus K^{\prime} \stackrel{\beta-i^{\prime}}{\longrightarrow} P^{\prime} \longrightarrow 0
$$

is a short exact sequence in $\mathfrak{V}$ ect, so we are left to show that this is actually a short exact sequence in $\mathfrak{D V}$ Vect. $(i, \alpha)$ is an induction since $i$ is. To show that $\left(\beta-i^{\prime}\right)$ is a subduction, note that there exists a smooth linear map $\delta^{\prime}: P^{\prime} \rightarrow K^{\prime}$ such that $\beta \circ \gamma-1_{P^{\prime}}=i^{\prime} \delta^{\prime}$. For any plot $p: U \rightarrow P^{\prime}$, the map $\left(\gamma \circ p, \delta^{\prime} \circ p\right): U \rightarrow P \oplus K^{\prime}$ is smooth, and we have $p=\left(\beta-i^{\prime}\right) \circ\left(\gamma \circ p, \delta^{\prime} \circ p\right)$. The conclusion then follows from Proposition $[\mathbf{L}](4)$.

Lemma 7.3 (Horseshoe). Let

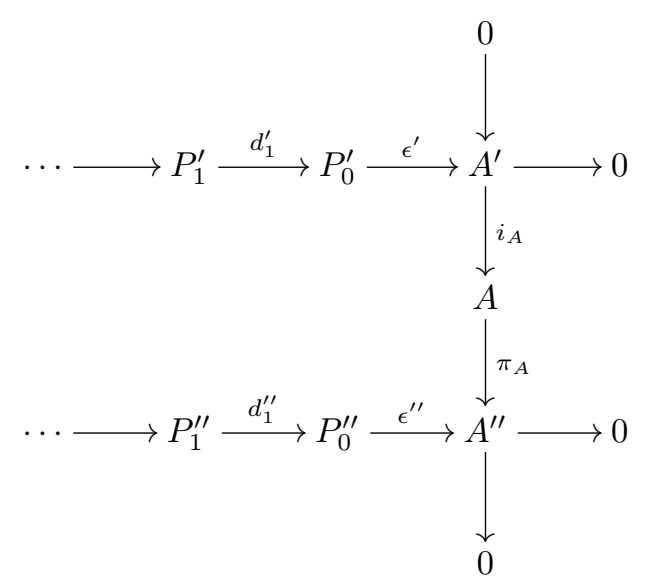

be a diagram in $\mathfrak{D V e c t}$ with the two horizontal lines diffeological projective resolutions of $A^{\prime}$ and $A^{\prime \prime}$ respectively, and the vertical line a short exact sequence of diffeological vector spaces. Then there exists a diffeological projective resolution of $A$ in the middle horizontal line making the following diagram commutative and all vertical lines short exact sequences in $\mathfrak{D V e c t :}$

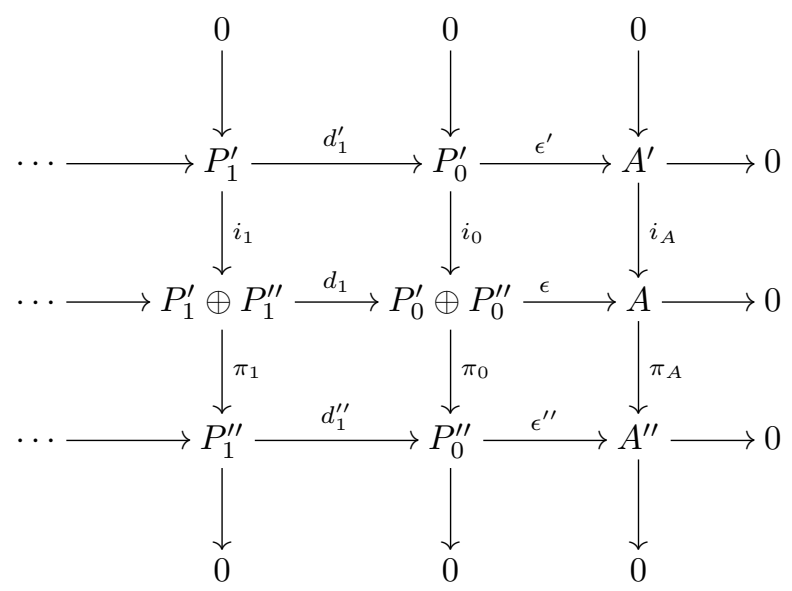

Proof. Since $\pi_{A}$ is a linear subduction and $P_{0}^{\prime \prime}$ is projective, there exists a smooth 
linear map $f: P_{0}^{\prime \prime} \rightarrow A$ such that $\pi_{A} \circ f=\epsilon^{\prime \prime}$. Define $\epsilon:=i_{A} \circ \epsilon^{\prime}+f$. The inductive proof of [W], Horseshoe Lemma 2.2.8] showed that

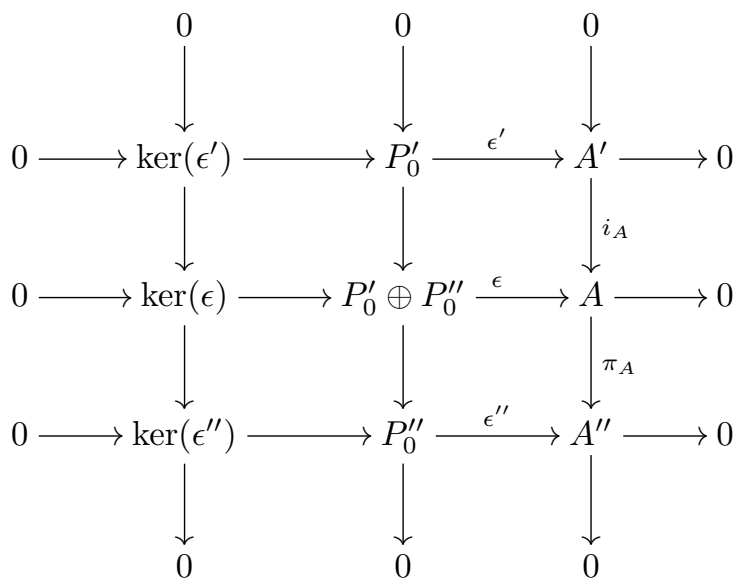

is a commutative diagram with all vertical and horizontal lines short exact sequences in $\mathfrak{V}$ ect, so we are left to show that $\epsilon: P_{0}:=P_{0}^{\prime} \oplus P_{0}^{\prime \prime} \rightarrow A$ is a subduction and $0 \rightarrow$ $\operatorname{ker}\left(\epsilon^{\prime}\right) \rightarrow \operatorname{ker}(\epsilon) \rightarrow \operatorname{ker}\left(\epsilon^{\prime \prime}\right) \rightarrow 0$ is a short exact sequence of diffeological vector spaces. Both of these follow from the fact that given plots $p: U \rightarrow P_{0}^{\prime \prime}$ and $q: U \rightarrow A$ such that $\epsilon^{\prime \prime} \circ p=\pi_{A} \circ q$, locally there exists a plot $r: U \rightarrow P_{0}$ such that $\epsilon \circ r=q$ and $\pi_{0} \circ r=p$. Since finite products and coproducts coincide in $\mathfrak{D V e c t}$, this fact follows from the assumptions that $P_{0}^{\prime}$ is a diffeological vector space, $\epsilon^{\prime}$ is a subduction, and $0 \rightarrow A^{\prime} \rightarrow A \rightarrow A^{\prime \prime} \rightarrow 0$ is a short exact sequence in $\mathfrak{D V e c t .}$

Theorem 7.4. Let $\boldsymbol{f}: \boldsymbol{A} \rightarrow \boldsymbol{B}$ and $\boldsymbol{g}: \boldsymbol{B} \rightarrow \boldsymbol{C}$ be diffeological chain maps between diffeological chain complexes, such that for each $n$,

$$
0 \longrightarrow A_{n} \stackrel{f_{n}}{\longrightarrow} B_{n} \stackrel{g_{n}}{\longrightarrow} C_{n} \longrightarrow 0
$$

is a short exact sequence in $\mathfrak{D V e c t . ~ T h e n ~ w e ~ h a v e ~ t h e ~ f o l l o w i n g ~ s e q u e n c e ~ i n ~} \mathfrak{D V e c t}$ which is exact in $\mathfrak{V}$ ect:

$$
\begin{gathered}
\cdots \longrightarrow H_{n}(\boldsymbol{A}) \stackrel{f_{*}}{\longrightarrow} H_{n}(\boldsymbol{B}) \stackrel{g_{*}}{\longrightarrow} H_{n}(\boldsymbol{C}) \\
\delta_{n} \longrightarrow H_{n-1}(\boldsymbol{A}) \stackrel{f_{*}}{\longrightarrow} H_{n-1}(\boldsymbol{B}) \stackrel{g_{*}}{\longrightarrow} \cdots
\end{gathered}
$$

Proof. Since every short exact sequence in $\mathfrak{D V e c t}$ is also a short exact sequence in $\mathfrak{V}$ ect, we have this long exact sequence in $\mathfrak{V e c t}$. By functoriality of homology functors, we know that all $f_{*}$ 's and $g_{*}$ 's are smooth. So we are left to show that all connecting linear maps $\delta$ are smooth. Recall that $\delta_{n}: H_{n}(\boldsymbol{C}) \rightarrow H_{n-1}(\boldsymbol{A})$ is defined as follows:

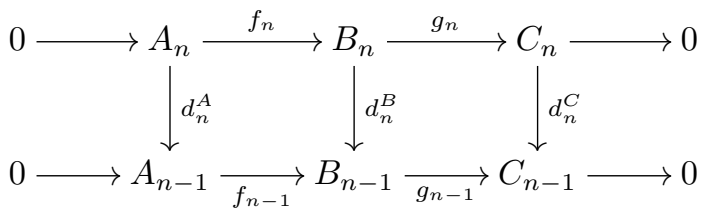


Pick a representative $c$ for $[c] \in \operatorname{ker}\left(d_{n}^{C}\right) / \operatorname{Im}\left(d_{n+1}^{C}\right)=H_{n}(\boldsymbol{C})$. Since $g_{n}$ is surjective, we can pick $b \in B_{n}$ such that $g_{n}(b)=c$. Then there exists $a \in \operatorname{ker}\left(d_{n-1}^{A}\right) \subseteq A_{n-1}$ such that $f_{n-1}(a)=d_{n}^{B}(b)$. The map $\delta_{n}$ is defined by $\delta_{n}([c])=[a]$, which is independent of the choice of any representative $c$ of $[c]$ and any lift $b \in B_{n}$. Any plot $p: U \rightarrow$ $H_{n}(\boldsymbol{C})$ globally lifts to a plot $q: U \rightarrow \operatorname{ker}\left(d_{n}^{C}\right)$ by Corollary $[$, since the quotient map $\operatorname{ker}\left(d_{n}^{C}\right) \rightarrow H_{n}(\boldsymbol{C})$ is a linear subduction. If we write $i: \operatorname{ker}\left(d_{n}^{C}\right) \rightarrow C_{n}$ for the inclusion map, then the plot $i \circ q: U \rightarrow C_{n}$ globally lifts to a plot $r: U \rightarrow B_{n}$ by Corollary 5 , since $g_{n}$ is a linear subduction. So by diagram chasing, the plot $d_{n}^{B} \circ$ $r: U \rightarrow B_{n-1}$ globally lifts to a plot $s: U \rightarrow \operatorname{ker}\left(d_{n-1}^{A}\right)$. This proves the smoothness of $\delta_{n}$.

Definition 7.5. Given diffeological vector spaces $V$ and $W$, we define

$$
\operatorname{Ext}^{n}(V, W):=H^{n}\left(L^{\infty}(\boldsymbol{P}(V), W)\right)
$$

where $\boldsymbol{P}(V) \rightarrow V$ is a diffeological projective resolution of $V$.

Here are some basic properties for $\operatorname{Ext}^{n}(V, W)$ :

\section{Proposition 7.6.}

1. $\operatorname{Ext}^{n}(V, W)$ does not depend on the choice of any diffeological projective resolution of $V$.

2. $\operatorname{Ext}^{0}(V, W)$ is always isomorphic to $L^{\infty}(V, W)$ in $\mathfrak{D}$ Vect.

3. If $V$ is projective, then $\operatorname{Ext}^{n}(V, W)=0$ for any diffeological vector space $W$ and any $n \geqslant 1$.

4. (Dimension shift) Let

$$
W \stackrel{d_{n}}{\longrightarrow} P_{n-1} \stackrel{d_{n-1}}{\longrightarrow} \cdots \longrightarrow P_{1} \stackrel{d_{1}}{\longrightarrow} P_{0} \stackrel{d_{0}}{\longrightarrow} V
$$

be a finite diffeological chain complex such that it is diffeologically exact at every middle spot, each $P_{i}$ is projective, $d_{0}$ is a linear subduction, and $d_{n}$ is a linear induction. Then $\operatorname{Ext}^{m+n}(V, A)$ is isomorphic to $\operatorname{Ext}^{m}(W, A)$ in $\mathfrak{D V e c t}$ for any $m \in \mathbb{Z}^{+}$and any diffeological vector space $A$.

5. Let $\left\{V_{i}\right\}_{i \in I} \cup\{W\}$ be a set of diffeological vector spaces. Then we have natural isomorphisms between $\operatorname{Ext}^{n}\left(\oplus_{i \in I} V_{i}, W\right)$ and $\prod_{i \in I} \operatorname{Ext}^{n}\left(V_{i}, W\right)$ in $\mathfrak{D V e c t}$ for all $n \in \mathbb{N}$.

6. Let $\{V\} \cup\left\{W_{j}\right\}_{j \in J}$ be a set of diffeological vector spaces. Then we have natural isomorphisms between $\operatorname{Ext}^{n}\left(V, \prod_{j \in J} W_{j}\right)$ and $\prod_{j \in J} \operatorname{Ext}^{n}\left(V, W_{j}\right)$ in $\mathfrak{D V e c t}$ for all $n \in \mathbb{N}$.

Proof. (1)-(4) are straightforward from definition.

To prove (5), besides the usual diagram chasing in homological algebra, we also need the following facts in $\mathfrak{D}$ Vect (and $\mathfrak{D}$ iff):

- Every (co)product of linear subductions is again a linear subduction. For the case of coproduct, it can be proved by the description of coproduct diffeology from the proof of Proposition [.2.

- Let $X_{j}$ be a subset of a diffeological space $Y_{j}$ for each $j \in J$. Then the subdiffeology on $\prod_{j \in J} X_{j}$ from $\prod_{j \in J} Y_{j}$ coincides with the product diffeology with each $X_{j}$ equipped with the sub-diffeology from $Y_{j}$. 
- Let $A_{i}$ be a linear subspace of $V_{i}$ for each $i \in I$. Then we have a natural isomorphism between $\left(\prod_{i \in I} V_{i}\right) /\left(\prod_{i \in I} A_{i}\right)$ and $\prod_{i \in I}\left(V_{i} / A_{i}\right)$ in $\mathfrak{D V e c t .}$

(6) can be proved similarly.

Remark 7.7. We can define injective diffeological vector space as dual of projective diffeological vector space. That is, a diffeological vector space $V$ is injective if and only if for every linear induction $f: W_{1} \rightarrow W_{2}$ and every smooth linear map $g: W_{1} \rightarrow V$, there exists a smooth linear map $h: W_{2} \rightarrow V$ such that $g=h \circ f$. It is straightforward to show that if $V$ is injective, then $\operatorname{Ext}^{n}(W, V)=0$ for any diffeological vector space $W$ and any $n \geqslant 1$. In particular, $\operatorname{Ext}^{n}\left(W, \mathbb{R}_{\text {ind }}\right)=0$ for any diffeological vector space $W$ and any $n \geqslant 1$. But I don't know any example of injective diffeological vector spaces other than indiscrete diffeological vector spaces.

Theorem 7.8. Let $0 \longrightarrow W_{1} \stackrel{i}{\longrightarrow} W_{2} \stackrel{\pi}{\longrightarrow} W_{3} \longrightarrow 0$ be a short exact sequence in $\mathfrak{D V e c t . ~ T h e n ~ f o r ~ a n y ~ d i f f e o l o g i c a l ~ v e c t o r ~ s p a c e ~} V$, we have the following sequence in $\mathfrak{D}$ Vect which is exact in $\mathfrak{V}$ ect:

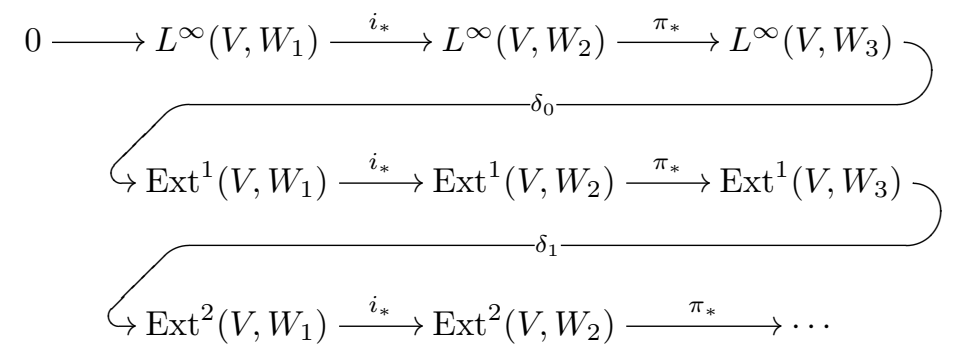

Proof. Take a diffeological projective resolution $\boldsymbol{P}(V) \rightarrow V$ of $V$. Since

$$
0 \longrightarrow W_{1} \stackrel{i}{\longrightarrow} W_{2} \stackrel{\pi}{\longrightarrow} W_{3} \longrightarrow 0
$$

is a short exact sequence in $\mathfrak{D V e c t}$ and each $P_{j}$ is projective, by Proposition $\mathbf{b}$. $\mathbf{b}$, we get diffeological cochain maps

$$
i_{*}: L^{\infty}\left(\boldsymbol{P}(V), W_{1}\right) \rightarrow L^{\infty}\left(\boldsymbol{P}(V), W_{2}\right)
$$

and

$$
\pi_{*}: L^{\infty}\left(\boldsymbol{P}(V), W_{2}\right) \rightarrow L^{\infty}\left(\boldsymbol{P}(V), W_{3}\right)
$$

between diffeological cochain complexes such that for each $n$

$$
0 \longrightarrow L^{\infty}\left(P_{n}, W_{1}\right) \stackrel{i_{*}}{\longrightarrow} L^{\infty}\left(P_{n}, W_{2}\right) \stackrel{\pi_{*}}{\longrightarrow} L^{\infty}\left(P_{n}, W_{3}\right) \longrightarrow 0
$$

is a short exact sequence in $\mathfrak{D V}$ Vect. The result then follows from the cohomological version of Theorem [..4.

Dually, we have

Theorem 7.9. Let $0 \longrightarrow V_{1} \stackrel{i}{\longrightarrow} V_{2} \stackrel{\pi}{\longrightarrow} V_{3} \longrightarrow 0$ be a short exact sequence in $\mathfrak{D V e c t . ~ T h e n ~ f o r ~ a n y ~ d i f f e o l o g i c a l ~ v e c t o r ~ s p a c e ~} W$, we have the following sequence 
in $\mathfrak{D}$ Vect which is exact in $\mathfrak{V}$ ect:

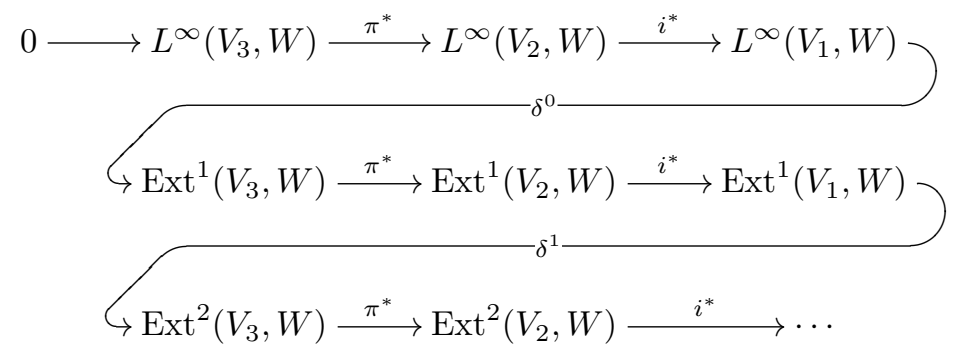

Proof. This follows directly from the Horseshoe Lemma and Theorem ㄷ.4.

Corollary 7.10. Let $V$ and $W$ be diffeological vector spaces. If $\operatorname{Ext}^{1}(W, V)=0$, then every short exact sequence $0 \rightarrow V \rightarrow A \rightarrow W \rightarrow 0$ in $\mathfrak{D}$ Vect splits smoothly.

Proof. By Theorem [r.8, we know that $L^{\infty}(W, A) \rightarrow L^{\infty}(W, W)$ is surjective. The result then follows from Theorem [.].

Hence, with the notations in Example $4.3, \operatorname{Ext}^{1}\left(\prod_{\omega} \mathbb{R}, K\right) \neq 0$.

Corollary 7.11. A diffeological vector space $V$ is projective if and only if

$$
\operatorname{Ext}^{1}(V, W)=0
$$

for every diffeological vector space $W$.

Proof. $(\Rightarrow)$ This follows from Proposition [.6(3).

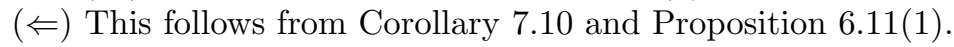

As an easy corollary, we know that if $V \rightarrow W$ is a linear subduction between projective diffeological vector spaces, then its kernel is also projective. In particular, if $f: M \rightarrow N$ is a smooth fiber bundle between smooth manifolds, then the kernel of $F(f): F(M) \rightarrow F(N)$ is a projective diffeological vector space.

Finally, we are going to prove the converse of Corollary 1 . We first show the diffeological version of the (Short) Five Lemma:

Lemma 7.12 (Short Five Lemma). Given a commutative diagram in $\mathfrak{D V e c t}$

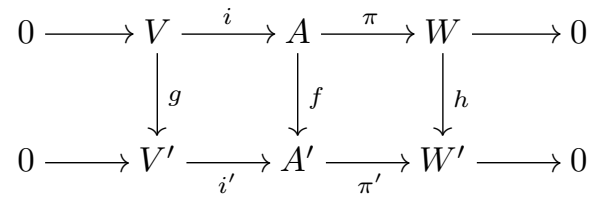

with both rows short exact sequences in $\mathfrak{D V e c t ,}$ and both $g$ and $h$ isomorphisms in $\mathfrak{D V e c t ,}$ then $f$ is also an isomorphism in $\mathfrak{D}$ Vect.

Proof. From [Hu, the Short Five Lemma 1.17], we know that $f$ is an isomorphism in Vect. We are left to show that $f^{-1}$ is smooth. For any plot $p: U \rightarrow A^{\prime}$, since $h^{-1} \circ \pi^{\prime} \circ p: U \rightarrow W$ is smooth and $\pi$ is a linear subduction, by Corollary we have a smooth map $q: U \rightarrow A$ such that $\pi \circ q=h^{-1} \circ \pi^{\prime} \circ p$. Then $\pi \circ\left(f^{-1} \circ p-q\right)=0$ 
implies that $\pi^{\prime} \circ(p-f \circ q)=0$. Since the bottom row of the above diagram is a short exact sequence in $\mathfrak{D V e c t}$, there exists a smooth map $r: U \rightarrow V^{\prime}$ such that $p-f \circ q=$ $i^{\prime} \circ r$. So $f^{-1} \circ p=q+f^{-1} \circ i^{\prime} \circ r=q+i \circ g^{-1} \circ r$ is smooth, which implies that $f^{-1}$ is smooth.

Slightly more generally, we can prove the following in a similar way:

Lemma 7.13 (Five Lemma). Let

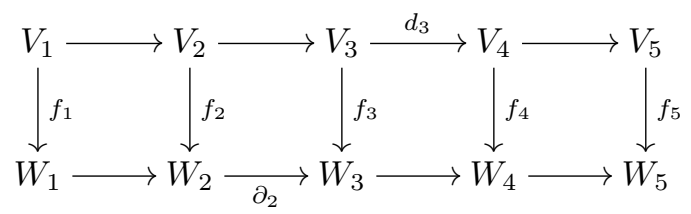

be a commutative diagram in $\mathfrak{D V e c t , ~ s u c h ~ t h a t ~ b o t h ~ r o w s ~ a r e ~ e x a c t ~ i n ~} \mathfrak{V}$ ect, $f_{1}$ is surjective, $f_{5}$ is injective, and both $f_{2}$ and $f_{4}$ are isomorphisms in DVect. If the induced map $V_{3} \rightarrow \operatorname{Im}\left(d_{3}\right)$ is a subduction and $W_{2} / \operatorname{ker}\left(\partial_{2}\right) \rightarrow W_{3}$ is an induction, then $f_{3}$ is an isomorphism in $\mathfrak{D}$ Vect.

For $V, W$ diffeological vector spaces, we define two short exact sequences $0 \rightarrow$ $V \rightarrow A \rightarrow W \rightarrow 0$ and $0 \rightarrow V \rightarrow A^{\prime} \rightarrow W \rightarrow 0$ in $\mathfrak{D V e c t}$ to be equivalent if there is a commutative diagram in $\mathfrak{D}$ Vect:

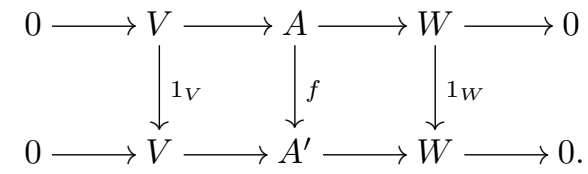

By the Short Five Lemma (Lemma $\mathbb{C}$ ), $f$ is an isomorphism in $\mathfrak{D}$ Vect. So this defines an equivalence relation on the set of all short exact sequences in $\mathfrak{D V e c t}$ of the form $0 \rightarrow V \rightarrow A \rightarrow W \rightarrow 0$, and we write the quotient set as $e(W, V)$.

Theorem 7.14. There is a bijection between $e(W, V)$ and $\operatorname{Ext}^{1}(W, V)$ which sends the class of smoothly split short exact sequences in $e(W, V)$ to 0 in $\operatorname{Ext}^{1}(W, V)$.

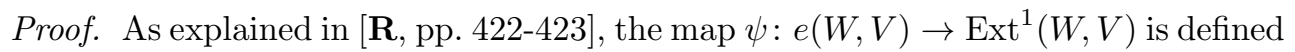
as follows: For any short exact sequence $0 \rightarrow V \rightarrow A \rightarrow W \rightarrow 0$ in $\mathfrak{D V e c t}$ and any diffeological projective resolution $\boldsymbol{P}(W) \rightarrow W$ of $W$, we have a commutative diagram (not necessarily unique) in $\mathfrak{D}$ Vect

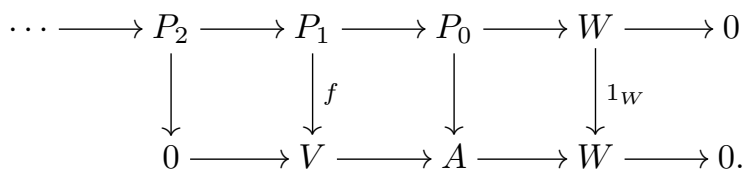

Then $\psi$ takes the class of $0 \rightarrow V \rightarrow A \rightarrow W \rightarrow 0$ in $e(W, V)$ to $[f] \in \operatorname{Ext}^{1}(W, V)$. As shown in $[\mathbb{R}]$, this map depends neither on the representing short exact sequence in $e(W, V)$ nor on the diffeological projective resolution of $W$ nor on the choice of $f$.

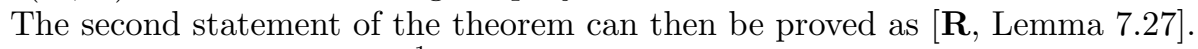

The inverse map $\theta: \operatorname{Ext}^{1}(W, V) \rightarrow e(W, V)$ can be constructed as follows: For any diffeological projective resolution $\boldsymbol{P}(W) \rightarrow W$ of $W$ and any $f \in L^{\infty}\left(P_{1}, V\right)$ with 


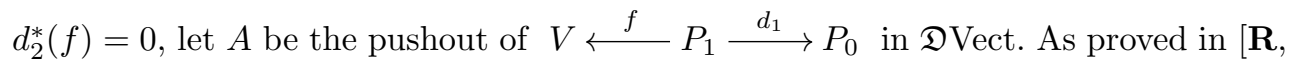
Lemma 7.28], we have a short exact sequence

$$
0 \longrightarrow V \stackrel{i}{\longrightarrow} A \stackrel{\pi}{\longrightarrow} W \longrightarrow 0
$$

in $\mathfrak{V}$ ect. It is easy to check that $\pi$ is a subduction since $P_{0} \rightarrow W$ is, and $i$ is an induction since $A$ is a pushout, $\operatorname{ker}\left(d_{0}\right) \rightarrow P_{0}$ is an induction, and $P_{1} \rightarrow \operatorname{ker}\left(d_{0}\right)$ is a subduction. As shown in $[\mathbb{R}$, Theorem 7.30], $\theta$ is independent of the choice of representative of $[f] \in \operatorname{Ext}^{1}(W, V)$, and $\theta$ is the inverse of $\psi$.

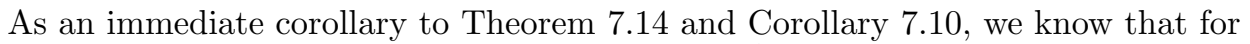
any fixed diffeological vector spaces $V$ and $W, \operatorname{Ext}^{1}(W, V)=0$ if and only if every short exact sequence $0 \rightarrow V \rightarrow A \rightarrow W \rightarrow 0$ in $\mathfrak{D}$ Vect splits smoothly.

\section{A model structure on diffeological chain complexes}

Following ideas from [CH] , we establish a cofibrantly generated model structure on the category $\mathfrak{D C h}$ of diffeological chain complexes, which resembles the projective model structure on the category $\mathfrak{C h}(R)$ of chain complexes of modules over a ring $R$ (see [H], Section 2.3]). Moreover, a diffeological projective resolution of a diffeological vector space is a cofibrant replacement of a canonical diffeological chain complex under this model structure, and Ext* can be expressed using the hom-functor in the corresponding homotopy category. Throughout this section, we follow the notations of [Ha, Chapter 2].

Let $V$ be a diffeological vector space. We write $S^{n}(V)$ for the diffeological chain complex with the only non-trivial diffeological vector space $V$ at the $n^{\text {th }}$ spot. We also write $D^{n}(V)$ for the diffeological chain complex with the only non-trivial diffeological vector spaces $V$ at the $n^{t h}$ and $(n-1)^{t h}$ spots, and differential $d_{n}=1_{V}$. There is a diffeological chain map $i_{n}^{V}: S^{n}(V) \rightarrow D^{n+1}(V)$ with $\left(i_{n}^{V}\right)_{n}=1_{V}$.

Let $I$ be the set of all diffeological chain maps $i_{n}^{F(U)}: S^{n}(F(U)) \rightarrow D^{n+1}(F(U))$ for all $n \in \mathbb{Z}$ and all open subsets $U$ of $\mathbb{R}^{m}$ for all $m \in \mathbb{N}$, let $J$ be the set of all diffeological chain maps $0 \rightarrow D^{n}(F(U))$ for all $n \in \mathbb{Z}$ and all open subsets $U$ of $\mathbb{R}^{m}$ for all $m \in \mathbb{N}$, and let $\mathcal{W}$ be the family of morphisms $\boldsymbol{f}: \boldsymbol{V} \rightarrow \boldsymbol{W}$ in $\mathfrak{D C h}$ such that $L^{\infty}(F(U), \boldsymbol{f}): L^{\infty}(F(U), \boldsymbol{V}) \rightarrow L^{\infty}(F(U), \boldsymbol{W})$ is a homology isomorphism in $\mathfrak{C h}(\mathbb{R})$ for all open subsets $U$ of $\mathbb{R}^{m}$ for all $m \in \mathbb{N}$.

The following two lemmas tell us more about $\mathcal{W}$ :

Lemma 8.1. Every $\boldsymbol{V} \rightarrow \boldsymbol{W}$ in $\mathcal{W}$ induces a diffeological homology isomorphism.

However, the converse is not true, i.e., not every diffeological homology isomorphism is in $\mathcal{W}$; see Remark $\mathbb{Z}$ for such an example.

Proof. Taking $U=\mathbb{R}^{0}$ in the definition of $\mathcal{W}$, we know that $\boldsymbol{f}: \boldsymbol{V} \rightarrow \boldsymbol{W}$ in $\mathcal{W}$ induces a smooth linear map $\left(f_{n}\right)_{*}: H_{n}(\boldsymbol{V}) \rightarrow H_{n}(\boldsymbol{W})$ for every $n$. So we are left to show that the inverse map is smooth. Write $Z_{n}(\boldsymbol{W})$ for $\operatorname{ker}\left(d_{n}^{\boldsymbol{W}}\right)$ equipped with the sub-diffeology of $W_{n}$. Since $\pi_{n}^{\boldsymbol{W}}: Z_{n}(\boldsymbol{W}) \rightarrow H_{n}(\boldsymbol{W})$ is a linear subduction, any plot $\alpha: U \rightarrow H_{n}(\boldsymbol{W})$ is of the form $\pi_{n}^{\boldsymbol{W}} \circ p$ for some plot $p: U \rightarrow Z_{n}(\boldsymbol{W})$. Since $\left(f_{n}\right)_{*}: H_{n}\left(C^{\infty}(U, \boldsymbol{V})\right) \rightarrow H_{n}\left(C^{\infty}(U, \boldsymbol{W})\right)$ is an isomorphism in $\mathfrak{V}$ ect, there exist plots 
$q: U \rightarrow Z_{n}(\boldsymbol{V})$ and $r: U \rightarrow W_{n+1}$ such that $f_{n} \circ q=p+d_{n+1}^{\boldsymbol{W}} \circ r:=\beta$. So we have a commutative diagram in $\mathfrak{D V}$ ect

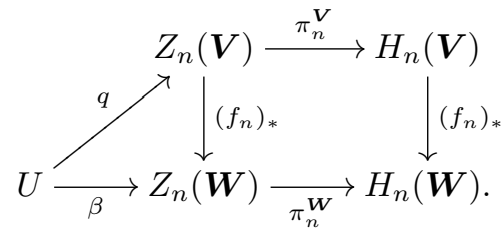

Note that $\pi_{n}^{\boldsymbol{W}} \circ \beta=\alpha$, so $\left(f_{n}\right)_{*}^{-1}: H_{n}(\boldsymbol{W}) \rightarrow H_{n}(\boldsymbol{V})$ is smooth.

Lemma 8.2. Let $\boldsymbol{A}$ be a diffeological chain complex. Then the following are equivalent:

1. $0 \rightarrow \boldsymbol{A}$ is in $\mathcal{W}$;

2. $d_{n+1}: A_{n+1} \rightarrow Z_{n}(\boldsymbol{A})$ is a linear subduction for each $n \in \mathbb{Z}$, where $Z_{n}(\boldsymbol{A})$ has the sub-diffeology of $A_{n}$;

3. $\boldsymbol{A}$ is diffeologically exact.

Proof. This is straightforward.

We are going to prove the following theorem in this section:

Theorem 8.3. The category $\mathfrak{D C h}$ of diffeological chain complexes and diffeological chain maps has a model structure with $I$ as its generating set of cofibrations, $J$ as its generating set of trivial cofibrations, and $\mathcal{W}$ as the family of its weak equivalences.

We will use the recognition theorem of cofibrantly generated model category (see, for example, [H], Theorem 2.1.19]) to prove this theorem.

Lemma 8.4. The category $\mathfrak{D C h}$ is complete and cocomplete.

Proof. Since the category $\mathfrak{D V e c t}$ is complete (Theorem $[$.$] ) and cocomplete (Theo-$

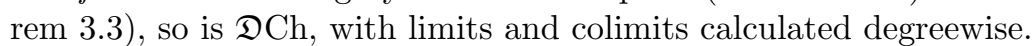

Since $\mathfrak{D V}$ Vect is an additive category with both kernels and cokernels (Remark $\mathbf{Z}$ ), so is $\mathfrak{D C h}$.

Corollary 8.5. Every diffeological chain complex is small in $\mathfrak{D C h}$.

Proof. Using the fact that the category $\mathfrak{D i f f}$ is locally presentable (see the proof of [CW7, Proposition 4.17]), one can show as [피, Example 2.1.6] that every diffeological vector space is small in $\mathfrak{D V e c t}$. The result then follows from a similar argument

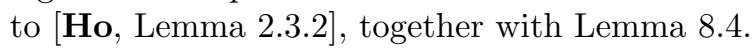

Lemma 8.6. $\mathcal{W}$ has the two out of three property and is closed under retracts.

Proof. This is straightforward.

Lemma 8.7. Let $\boldsymbol{f}: \boldsymbol{V} \rightarrow \boldsymbol{W}$ be a morphism in $\mathfrak{D C h}$. Then 
1. a square

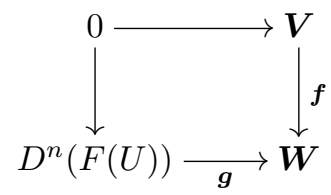

is in $\mathfrak{D C h}$ if and only if $g_{n} \circ i_{U}: U \rightarrow W_{n}$ is smooth; a lift $\boldsymbol{h}: D^{n}(F(U)) \rightarrow \boldsymbol{V}$ of the above square exists in $\mathfrak{D C h}$ if and only if we have a commutative triangle in $\mathfrak{D i f f}$

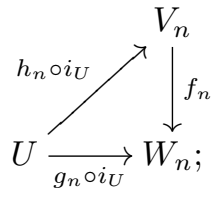

2. a square

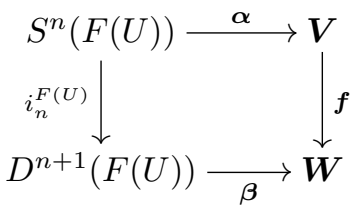

is commutative in $\mathfrak{D C h}$ if and only if the maps $\alpha_{n} \circ i_{U}: U \rightarrow V_{n}$ and $\beta_{n+1} \circ$ $i_{U}: U \rightarrow W_{n+1}$ are smooth, $\alpha_{n} \circ i_{U}$ has image in $Z_{n}(\boldsymbol{V})$, and $d_{n+1}^{\boldsymbol{W}} \circ \beta_{n+1} \circ$ $i_{U}=f_{n} \circ \alpha_{n} \circ i_{U}$; a lift $\gamma: D^{n+1}(F(U)) \rightarrow \boldsymbol{V}$ of the above commutative square exists in $\mathfrak{D C h}$ if and only if the dotted arrow exists so that the following two triangles commute in Diff:

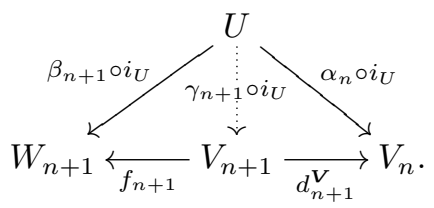

Proof. This follows directly from diagram chasing and the universal property of free diffeological vector space generated by a diffeological space.

Lemma 8.8. A morphism $\boldsymbol{f}: \boldsymbol{V} \rightarrow \boldsymbol{W}$ in $\mathfrak{D C h}$ is in $J$-inj if and only if $f_{n}: V_{n} \rightarrow W_{n}$ is a subduction for every $n \in \mathbb{Z}$.

Proof. This follows from Lemma 1 . (1) and the universal property of free diffeological vector space generated by a diffeological space.

Proposition 8.9. A morphism $\boldsymbol{f}: \boldsymbol{V} \rightarrow \boldsymbol{W}$ in $\mathfrak{D C h}$ is in I-inj if and only if it is in both J-inj and $\mathcal{W}$.

Proof. $(\Rightarrow)$ We first show that $\boldsymbol{f}$ is in $J$-inj, i.e., $f_{n}: V_{n} \rightarrow W_{n}$ is a subduction for every $n \in \mathbb{Z}$ by Lemma 8 . Here is the proof. For any plot $p: U \rightarrow W_{n}$, we have a 
commutative square in $\mathfrak{D i f f :}$

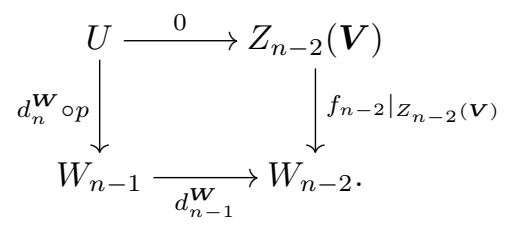

By Lemma $\square .(2)$, there exists a plot $q: U \rightarrow V_{n-1}$ making the following diagram commutative in $\mathfrak{D}$ iff:

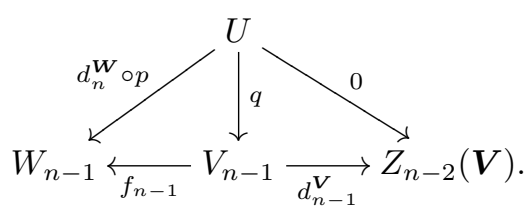

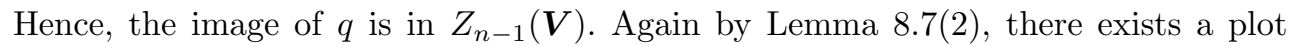
$r: U \rightarrow V_{n}$ making the following diagram commutative in $\mathfrak{D i f f :}$

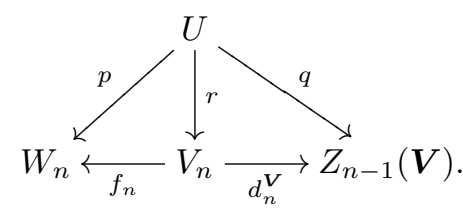

Therefore, $f_{n}: V_{n} \rightarrow W_{n}$ is a subduction for every $n \in \mathbb{Z}$.

Now we show that $f$ is in $\mathcal{W}$. Here is the proof. By functoriality, there is a natural map $H_{n}(U, \boldsymbol{f}): H_{n}\left(C^{\infty}(U, \boldsymbol{V})\right) \rightarrow H_{n}\left(C^{\infty}(U, \boldsymbol{W})\right)$ for each $n \in \mathbb{Z}$. We are left to show that they are bijections.

For surjectivity, note that a representative of an element in $H_{n}\left(C^{\infty}(U, \boldsymbol{W})\right)$ is a plot $a: U \rightarrow W_{n}$ such that $d_{n}^{W} \circ a=0$. So we have a commutative square in $\mathfrak{D}$ iff

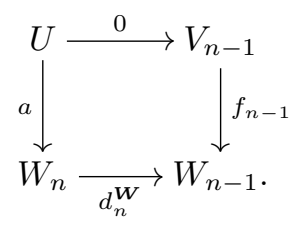

By Lemma $\square .(2)$, there exists a plot $b: U \rightarrow V_{n}$ making the following diagram commutative in $\mathfrak{D}$ iff:

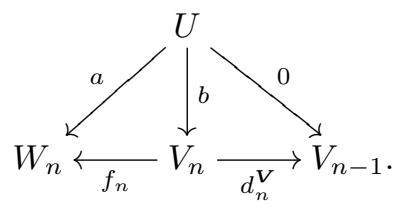

Therefore, the map $H_{n}(U, \boldsymbol{f})$ is surjective.

For injectivity, note that if a representative $c$ of an element in $H_{n}\left(C^{\infty}(U, \boldsymbol{V})\right)$ is mapped to [0] in $H_{n}\left(C^{\infty}(U, \boldsymbol{W})\right)$ by $H_{n}(U, \boldsymbol{f})$, then $c: U \rightarrow V_{n}$ is smooth with image 
in $Z_{n}(\boldsymbol{V})$, and there exists a plot $d: U \rightarrow W_{n+1}$ such that the following square is commutative in $\mathfrak{D i f f}:$

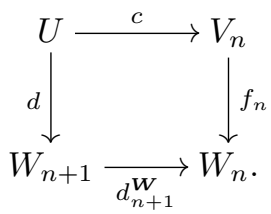

By Lemma $\square .(2)$, there exists a plot $e: U \rightarrow V_{n+1}$ making the following diagram commutative in $\mathfrak{D}$ iff:

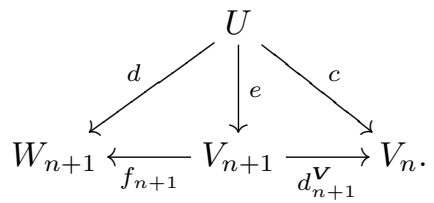

Therefore, $[c]=[0] \in H_{n}\left(C^{\infty}(U, \boldsymbol{V})\right)$, and the map $H_{n}(U, \boldsymbol{f})$ is injective.

$(\Leftarrow)$ By Lemma $\mathbb{E .}(2)$, we are left to show that for any commutative square in Diff

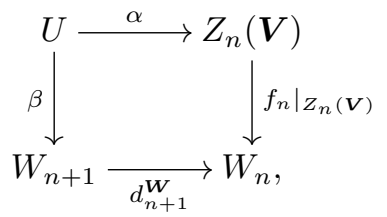

we can find a plot $\gamma: U \rightarrow V_{n+1}$ making the following diagram commutative in $\mathfrak{D}$ iff:

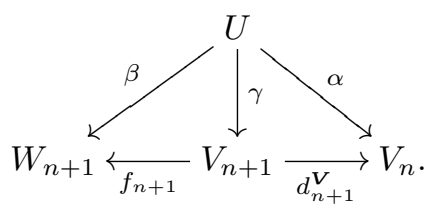

Since $f_{n+1}: V_{n+1} \rightarrow W_{n+1}$ is a linear subduction, by Corollary 5 there exists a plot $\gamma^{\prime}: U \rightarrow V_{n+1}$ such that $\beta=f_{n+1} \circ \gamma^{\prime}$. One checks that $f_{n} \circ\left(\alpha-d_{n+1}^{V} \circ \gamma^{\prime}\right)=0$ and $d_{n}^{V} \circ\left(\alpha-d_{n+1}^{V} \circ \gamma^{\prime}\right)=0$. Hence, $\left[\alpha-d_{n+1}^{V} \circ \gamma^{\prime}\right] \in H_{n}\left(C^{\infty}(U, \boldsymbol{V})\right)$ and $H_{n}(U, \boldsymbol{f})$ sends this class to $[0] \in H_{n}\left(C^{\infty}(U, \boldsymbol{W})\right)$. By the assumption that $H_{n}(U, \boldsymbol{f})$ is a bijection, there exists a plot $\gamma^{\prime \prime}: U \rightarrow V_{n+1}$ such that $d_{n+1}^{V} \circ \gamma^{\prime \prime}=\alpha-d_{n+1}^{V} \circ \gamma^{\prime}$. So $d_{n+1}^{\boldsymbol{W}} \circ f_{n+1} \circ \gamma^{\prime \prime}=0$, i.e., $\left[f_{n+1} \circ \gamma^{\prime \prime}\right] \in H_{n+1}\left(C^{\infty}(U, \boldsymbol{W})\right)$. By the assumption that $H_{n+1}(U, \boldsymbol{f})$ is a bijection, there exist plots $\gamma^{\prime \prime \prime}: U \rightarrow V_{n+1}$ and $\theta: U \rightarrow W_{n+2}$ such that $f_{n+1} \circ \gamma^{\prime \prime \prime}=f_{n+1} \circ \gamma^{\prime \prime}+d_{n+2}^{W} \circ \theta$ and $d_{n+1}^{V} \circ \gamma^{\prime \prime \prime}=0$. By the assumption that $f_{n+2}: V_{n+2} \rightarrow W_{n+2}$ is a linear subduction, there exists a plot $\rho: U \rightarrow V_{n+2}$ such that $\theta=f_{n+2} \circ \rho$. Then $\gamma:=\gamma^{\prime}+\gamma^{\prime \prime}-\gamma^{\prime \prime \prime}+d_{n+2}^{V} \circ \rho$ has the desired property.

Lemma 8.10. If $0 \rightarrow \boldsymbol{A}$ is in $J$-cof, then it is in $\mathcal{W}$.

Proof. Write $C(\boldsymbol{A})$ for the mapping cone of the identity map $1_{\boldsymbol{A}}: \boldsymbol{A} \rightarrow \boldsymbol{A}$. That is, $(C(\boldsymbol{A}))_{n}=A_{n} \oplus A_{n+1}$, and $d_{n}^{C(\boldsymbol{A})}(x, y)=\left(d_{n}^{\boldsymbol{A}}(x), x-d_{n+1}^{\boldsymbol{A}}(y)\right)$. Then $\boldsymbol{\pi}: C(\boldsymbol{A}) \rightarrow \boldsymbol{A}$ defined by $\pi_{n}(x, y)=x$ is a diffeological chain map, and hence each $\pi_{n}$ is a subduction, 
i.e., $\boldsymbol{\pi}$ is in $J$-inj by Lemma Since $0 \rightarrow \boldsymbol{A}$ is in $J$-cof, we have a commutative diagram in $\mathfrak{D C h}$

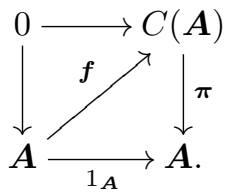

So $0 \rightarrow \boldsymbol{A}$ is a retract of $0 \rightarrow C(\boldsymbol{A})$. By Lemma $\mathbf{D}$, it is enough to show that $0 \rightarrow$ $C(\boldsymbol{A})$ is in $\mathcal{W}$. For any open subset $U$ of a Euclidean space, one can easily check that if $(p, q): U \rightarrow A_{n} \oplus A_{n+1}$ is a plot such that $d_{n}^{C(\boldsymbol{A})} \circ(p, q)=0$, then $(q, 0): U \rightarrow$ $A_{n+1} \oplus A_{n+2}$ is a plot such that $d_{n+1}^{C(\boldsymbol{A})} \circ(q, 0)=(p, q)$, which is desired.

Proposition 8.11. If $\boldsymbol{f}: \boldsymbol{A} \rightarrow \boldsymbol{B}$ is in $J$-cof, then it is in $\mathcal{W}$.

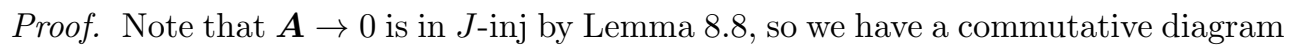
in $\mathfrak{D C h}$

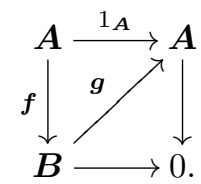

Therefore, each $f_{n}: A_{n} \rightarrow B_{n}$ is a linear induction. Let $\boldsymbol{C}$ be the cokernel of $\boldsymbol{f}$ in $\mathfrak{D C h}$. Then $0 \rightarrow \boldsymbol{C}$ is in $J$-cof, and for each $n \in \mathbb{Z}$,

$$
0 \longrightarrow A_{n} \stackrel{f_{n}}{\longrightarrow} B_{n} \longrightarrow C_{n} \longrightarrow 0
$$

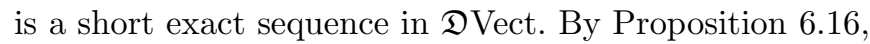

$$
0 \longrightarrow C^{\infty}\left(U, A_{n}\right) \stackrel{\left(f_{n}\right)_{*}}{\longrightarrow} C^{\infty}\left(U, B_{n}\right) \longrightarrow C^{\infty}\left(U, C_{n}\right) \longrightarrow 0
$$

is also a short exact sequence in $\mathfrak{D V}$ ect for any open subset $U$ of a Euclidean space and any $n$. Theorem

Proof of Theorem [8.3. By the recognition theorem of cofibrantly generated model category (see [ $\mathbb{H} \boldsymbol{d}$, Theorem 2.1.19] for instance), Theorem $\mathbf{B}]$ then follows from

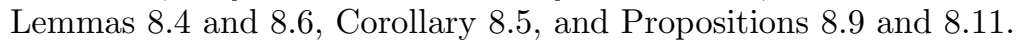

Now we establish several lemmas to understand elements in $I$-cof.

Lemma 8.12. Let $\boldsymbol{f}: \boldsymbol{V} \rightarrow \boldsymbol{W}$ be in I-inj, and let $\boldsymbol{K}$ be its kernel in $\mathfrak{D C h}$. Then $\boldsymbol{K}$ is diffeologically exact.

Proof. By Proposition 8.9 and Lemma 8.8 , we know that each $f_{n}$ is a linear subduction and $\boldsymbol{f}$ is in $\mathcal{W}$. So by Lemma $\square$,

$$
0 \longrightarrow K_{n} \longleftrightarrow V_{n} \stackrel{f_{n}}{\longrightarrow} W_{n} \longrightarrow 0
$$

is a short exact sequence in $\mathfrak{D V}$ Vect for each $n$. By Proposition $\left[\begin{array}{l}6 \\ \text {, }\end{array}\right.$

$$
0 \longrightarrow C^{\infty}\left(U, K_{n}\right) \longrightarrow C^{\infty}\left(U, V_{n}\right) \stackrel{\left(f_{n}\right)_{*}}{\longrightarrow} C^{\infty}\left(U, W_{n}\right) \longrightarrow 0
$$

is also a short exact sequence in $\mathfrak{D V e c t}$ for any open subset $U$ of a Euclidean space. 
Since $\boldsymbol{f}$ is in $\mathcal{W}$, by Theorem $\mathbb{C}, C^{\infty}(U, \boldsymbol{K})$ is exact, i.e., $0 \rightarrow \boldsymbol{K}$ is in $\mathcal{W}$. The result then follows from Lemma 8.2 .

Lemma 8.13. If $0 \rightarrow \boldsymbol{A}$ is in I-cof, then each $A_{n}$ is a projective diffeological vector space. Conversely, if $\boldsymbol{A}$ is a bounded below diffeological chain complex such that each $A_{n}$ is a projective diffeological vector space, then $0 \rightarrow \boldsymbol{A}$ is in I-cof.

Proof. This follows from a similar proof as for [H, Lemma 2.3.6] together with Lemma 8.2 .

Therefore, a diffeological projective resolution of a diffeological vector space $V$ is a cofibrant replacement of the diffeological chain complex $S^{0}(V)$ in this model structure on $\mathfrak{D C h}$.

\section{Proposition 8.14.}

1. Let $\boldsymbol{C}$ and $\boldsymbol{K}$ be diffeological chain complexes such that $0 \rightarrow \boldsymbol{C}$ is in $I$-cof and $\boldsymbol{K}$ is diffeologically exact. Then every diffeological chain map $\boldsymbol{C} \rightarrow \boldsymbol{K}$ is diffeologically chain homotopic to the zero map.

2. Let $\boldsymbol{f}: \boldsymbol{V} \rightarrow \boldsymbol{W}$ be a diffeological chain map with cokernel $\boldsymbol{C}$. Then $\boldsymbol{f}$ is in $\boldsymbol{I}$-cof if and only if it is a degreewise linear induction and $0 \rightarrow \boldsymbol{C}$ is in I-cof.

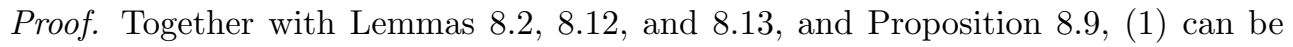

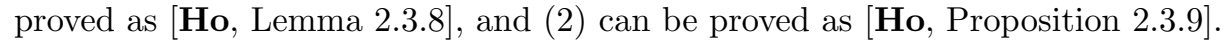

Write $\mathrm{Ho}(\mathfrak{D C h})$ for the homotopy category of $\mathfrak{D C h}$, i.e., the localization of $\mathfrak{D C h}$ with respect to the weak equivalences $\mathcal{W}$. By a similar proof as for [DS, Proposition 7.3], we have:

Proposition 8.15. Let $V$ and $W$ be diffeological vector spaces, and let $m$ and $n$ be natural numbers. Then $\mathrm{Ho}(\mathfrak{D C h})\left(S^{m}(V), S^{n}(W)\right)=\mathrm{Ext}^{n-m}(V, W)$.

\section{References}

[BH] J.C. Baez and A.E. Hoffnung, Convenient categories of smooth spaces, Trans. Amer. Math. Soc. 363(11), 5789-5825 (2011).

[CG] K. Costello and O. Gwilliam, Factorization Algebras in Quantum Field Theory, preprint available at http://math.northwestern.edu/ costello/ Eactorization.pdt.

[CH] J.D. Christensen and M. Hovey, Quillen model structures for relative homological algebra, Math. Proc. Cambridge Philos. Soc. 133(2), 261-293 (2002).

[CSW] J.D. Christensen, G. Sinnamon, and E. Wu, The $D$-topology for diffeological spaces, Pacific Journal of Mathematics 272(1), 87-110 (2014).

[CW1] J.D. Christensen and E. Wu, The homotopy theory of diffeological spaces, New York Journal of Mathematics 20, 1269-1303 (2014).

[CW2] J.D. Christensen and E. Wu, Tangent spaces and tangent bundles for diffeological spaces, preprint available at http://arxiv.org/abs/1411.5425.

[DS] W.G. Dwyer and J. Splinski, Homotopy theories and model categories, Handbook of algebraic topology, 73-126, North-Holland, Amsterdam, 1995. 
[Ho] M. Hovey, Model Categories, Mathematical Surveys and Monographs 63, AMS (1999).

[Hu] T.W. Hungerford, Algebra, Graduate Texts in Mathematics 73, Springer (1974).

[I1] P. Iglesias-Zemmour, Diffeology of the infinite Hopf fibration, Geometry and topology of manifolds, Banach Center Publ., Polish Acad. Sci. Inst. Math. 76, 349-393, Warsaw, (2007).

[I2] P. Iglesias-Zemmour, Dimension in diffeology, Indag. Math. 18(4), 555-560 (2007).

[I3] P. Iglesias-Zemmour, Diffeology, Mathematical Surveys and Monographs 185, AMS (2013).

[KM] A. Kriegl and P.W. Michor, The Convenient Setting of Global Analysis, Mathematical Surveys and Monographs 53, AMS (1997).

[M] S. MacLane, Categories for the Working Mathematician, Graduate Texts in Mathematics 5, second edition, Springer (1998).

[R] J.J. Rotman, An Introduction to Homological Algebra, Universitext, second edition, Springer (2009).

[Sc] J.P. Schneiders, Quasi-abelian categories and sheaves, Mém. Soc. Math. Fr. (N.S.) 76, (1999). Available at: http://www.analg.ulg.ac.be/jps/rec/ gacs.pdf.

[Se] R.T. Seeley, Extension of $C^{\infty}$ functions defined in a half space, Proc. Amer. Math. Soc. 15, 625-626 (1964).

[So1] J.M. Souriau, Groupes différentiels, Differential geometrical methods in mathematical physics (Proc. Conf., Aix-en-Provence/Salamanca, 1979), Lecture Notes in Math. 836, Springer, 91-128 (1980).

[So2] J.M. Souriau, Groupes différentiels de physique mathématique, South Rhone seminar on geometry, II (Lyon, 1983), Travaux en Cours, Hermann, Paris, 73-119 (1984).

[St] A. Stacey, Comparative smootheology, Theory and Applications of Categories 25(4), 64-117 (2011).

[V] M. Vincent, Diffeological differential geometry, Masters Thesis, University of Copenhagen, Denmark (2008).

[We] C.A. Weibel, An Introduction to Homological Algebra, Cambridge University Press (1997).

[Wu E. Wu, A homotopy theory for diffeological spaces, Ph.D. Thesis, the University of Western Ontario, Canada (2012).

Enxin $\mathrm{Wu}$ enxin.wudunivie.ac.at

DIANA Group, Faculty of Mathematics, University of Vienna, Oskar-MorgensternPlatz 1, 1090 Vienna, Austria 